\title{
REVISION OF NYMPHAEA CANDIDA RANGE - NEW DATA ON THE DISTRIBUTION AND HABITAT PREFERENCES OF THE SPECIES IN SOUTHERN POLAND
}

\author{
ARKADIUSZ NOWAK ${ }^{1}$, MARCIN NOBIS ${ }^{2}$, ZYGMUNT DAJDOK ${ }^{3}$, JOANNA ZALEWSKA-GAŁOSZ ${ }^{2}$, \\ SYlWIA NOWAK ${ }^{1}$, AGNIESZKA NOBIS ${ }^{2}$, IZABELA CZERNIAWSKA-KUSZA ${ }^{4}$, MACIEJ KOZAK ${ }^{5}$, \\ Adam Stebel ${ }^{6}$, Renata Bula ${ }^{7}$, Piotr Sugier $^{8}$, Andrzej SzlachetKA ${ }^{9}$, WAldeMAr BenA ${ }^{10}$, \\ AnNA TROJECKA ${ }^{2}$, RENATA PIWOWARCZYK ${ }^{11}$, AlEKSANDRA AdAMIEC ${ }^{2}$, RAFAŁ KraWCZYK ${ }^{12}$ \\ ${ }^{1}$ Laboratory of Geobotany and Plant Conservation, \\ Department of Biosystematics, Opole University \\ Oleska 48, 45-022 Opole, Poland \\ e-mail: anowak@uni.opole.pl \\ 2 Department of Plant Taxonomy and Phytogeography, \\ Institute of Botany, Jagiellonian University \\ ${ }^{5}$ Department of Plant Ecology, Jagiellonian University \\ Kopernika 27, 31-501 Kraków, Poland \\ ${ }^{3}$ Institute of Plant Biology Wrocław University \\ Kanonia 6/8, 50-328 Wrocław, Poland \\ ${ }^{4}$ Department of Land Cover Protection, \\ Opole University \\ Oleska 22, 45-052 Opole, Poland \\ ${ }^{6}$ Department of Pharmaceutical Botany, \\ Medical University of Katowice \\ Ostrogórska 30, 41-200 Sosnowiec, Poland \\ ${ }^{7}$ Centre of Nature Heritage of the Upper Silesia \\ św. Huberta 1, 40-543 Katowice, Poland \\ ${ }^{8}$ Department of Ecology, Institute of Biology \\ 12 Department of Nature Conservation \\ Maria Curie-Skłodowska University \\ Akademicka 19, 20-033 Lublin, Poland \\ ${ }^{9}$ Parszowice 81, 59-300 Ścinawa, Poland \\ 10 Olszewskiego 7, 59-900 Zgorzelec, Poland \\ ${ }^{11}$ Department of Botany, Institute of Biology \\ Jan Kochanowski University \\ Świętokrzyska 15, 25-406 Kielce, Poland
}

(Received: January 8, 2010. Accepted: May 12, 2010)

\section{ABSTRACT}

The paper presents results of geobotanical and taxonomic studies on the distribution and habitat requirements of Nymphaea candida in southern Poland. The researches were conducted in southern Poland in 2003-2009, in the provinces of Lower Silesia, Lublin, Małopolska, Opole, Silesian province as well as, in southern parts of Mazowieckie and Lubuskie. Flowers, leaves and fruits of Nymphaea species were collected from 27 locations. Altogether pollens from 73 populations of $N$. candida and 18 of $N$. alba from all the researched area were measured. The trophic level of an ecosystem was evaluated according to the results of the total nitrogen, total phosphorus, chlorophyll $a$, transparency and biological parameters.

As the result of the studies of more than 200 water bodies, 57 localities of $N$. candida were documented within the investigation area. The populations of $N$. candida occupy mid-forest water bodies and river ox-bow lakes. A 
significant number of populations was also found in artificial reservoirs - fish ponds. The most suitable habitat conditions for $N$. candida occur in shallow waters in the shore zone with the amplitude of the water column vary from 0.5 to $2 \mathrm{~m}$. Regarding the trophy level, $N$. candida occupies different habitats, mainly mesotrophic and also eutrophic with high content of organic matters. Considering the 15 checked morphological parameters, especially the stigma diameter, the number of carpellary teeth, flower and pollen diameters, the found and collected specimens of $N$. candida significantly differ from $N$. alba.

The study confirms that $N$. candida ocurrs in whole lowland Poland without any regional distribution gaps. According to the IUCN guidelines to species assessment the data gathered during the presented study do not allow to classify $N$. candida as a vulnerable species in Poland. Still existing populations for more than 150 years, numerous stable locations, abundant populations, a habitat accessibility, a biotope extent, an ecological amplitude against the trophy level and direct human impacts suggest, that the species should be regarded as a least concern (LC) taxon.

KEY WORDS: Nymphaea candida, distribution, ponds, aquatic vegetation, habitat, range.

\section{INTRODUCTION}

Nymphaea candida C. Presl. is an Euro-Siberian element of aquatic flora with the western range limit on the line of the Rhine (Hegi 1965; Meusel et al. 1965; Hulten and Fries 1986; Muntendam et al. 1996). According to Wayda (2000), N. candida occurs in Poland only in the northern part of the country reaching there the southern limit of its general continuous range. However, the species was also reported from the Czech Republic (Hejný and Slavík 1997), southern regions of Germany (Benkert et al. 1996), eastern France, Switzerland, south-western Romania, Austria, Hungary and former Yugoslavia (Tutin et al. 2002; Muntendam et al. 1996). The general distribution map of the species in Europe is presented in Fig. 1. Considering the fact that aquatic plants are distributed widely and limited mostly by climatic zones, a distribution gap in southern Poland is hardly possible to really exist. Moreover, N. candida was reported from southern Poland by Polish and German botanists in the 19th and early 20th centuries (detailed list of papers cited in Results). Unfortunately, the herbarium materials documented these localities and deposited in WRSL were destroyed during the Second World War, so they could not be re-examined. It is worth mentioning that the data published by German botanists were based on careful plant examinations. Moreover, most of their floristic reports have been later confirmed, so they are regarded as a credible source of botanical information. Nevertheless, these data were questioned by Wayda (2000), and in result not included in the "Atlas of Vascular Plants of Poland" (Zając and Zając 2001). On the other hand, the occurrence of $N$. candida in the area of Silesia and the Pojezierze Łęczyńsko-Włodawskie Lakeland was mentioned by Kłosowski (2001) in the "Polish Red Data Book of Plants". Localities of the species were also reported from the southern part of Lubuskie province by KujawaPawlaczyk and Pawlaczyk (2003) and from Lower Silesia by Bobrowicz and Konieczny (2000). Authors of the present paper have found the individuals of water lily in southern Poland, identified in the field as $N$. candida, based on the botanical key of Rutkowski (1998) or Kubát (2002). Further investigations proved, that $N$. candida occurs also in other regions of central and southern Poland (Nobis 2007; Nowak and Nowak 2007).

\section{RECOGNITION}

$N$. candida is closely related to $N$. alba L., with many varieties and subspecies described (Glück 1924; Heukels and Van der Meijden 1990). Despite the fact that the species has been extensively investigated (Glück 1924; Neuhäusl and Tomšovic 1957; Radics 1967; Casper and Krausch 1981; Jones and Clarke 1981; Muntendam et al. 1996), its identification still brings confusions. Most useful for identification both of the species during the field studies is the number of stigma rays. In the case of $N$. candida, the stigma consists of 9-14 rays, whereas in the case of $N$. alba between 15 and 25 (Muntendam et al. 1996). Several specimens with 12-13 stigma rays were hardly possible to determine according to pollen diameter or exine shape. Surelly these cases are rather hybrids of both species. The individuals collected by the authors of this paper in southern Poland have usually had 9 or even 7 carpellary teeth in stigma. Also the equatorial diameter of pollen grains seems to be a comfortable feature in some cases, however these analyses are possible only in the laboratory. The highest values of the equatorial length of $N$. alba pollen do not exceed $42 \mu \mathrm{m}$. The pollen of $N$. candida are in general larger than those of N. alba (Muntendam et al. 1996).

The morphologically closest to $N$. candida is $N$. alba var. minor DC. It is a starvation form differing from typical specimens of Nymphaea alba only in size. Recently, this form has been reported from Opole Silesia (Spałek 2007), but with no taxonomical evidences and herbarium documentation. According to Oberdorfer (1994) and Muntendam et al. (1996), the occurrence of N. alba var. minor is restricted to colder, northern regions of Europe.

A separate taxonomical problem related with Nymphaea candida is the possible hybridisation with N. alba. Morphologically intermediate forms between these two species are known for a quite long time (e.g. Conard 1905; Glück 1924). Also during N. candida survey in southern Poland such intermediate forms were collected. Molecular studies on hybrid specimens of $N$. alba and N. candida are planed in the future.

The Nymphaea genus is also of considerable importance from the conservation point of view. Both species are legally protected in Poland (Rozporządzenie Ministra Srodowiska 2004). As a taxon with restricted range in 
Poland $N$. candida was categorised as a vulnerable species in the Polish Red Data Book of Vascular Plants (Kłosowski 2001). So, it is important to have precise information about the distribution of both species to conduct the effective management and conservation.

The successive aims of the present study were: 1) to provide old, published floristic data on the distribution of $N$. candida in southern Poland; 2) to present the current distribution of the species in the discussed area; 3 ) to stimulate further molecular researches on infraspecific hybridisation in the genus in the area of co-occurrence of the two species representing the genus Nymphaea; 4) to provide data on habitat preferences of $N$. candida from selected localities, and finally 5) to discuss the present threat status of $N$. candida in Poland.

\section{MATERIALS AND METHODS}

The field studies were conducted in southern Poland in 2003-2009 (Figs 1 and 2). Flowers, leaves and fruits of the Nymphaea species were collected from 27 locations, mainly in Opole Silesia in July and September 2008 on the allowance DKFOPPogiz-4211/I-62/1887/08/ep issued by the Polish Minister of Environment. The plants were collected during warm days between $10 \mathrm{am}$. and $6 \mathrm{pm}$. The fresh material was identified and described in the field using the botanical key of Rutkowski (1998).

On the basis of botanical keys by Rutkowski (1998), Kubát (2002), Oberdorfer (1994), Hejný and Slavík (1997), as well as regarding the research works of Muntendam et al. (1996) and Wayda (2000), the following fifteen features were chosen to describe the specimens: the diameter of the stigma (column 1 in Table 1), the number of carpellary teeth (2), the position of the flower on water (3), the shape of the flower (4), the side view of the flowerbase (5), the underside view of the flowerbase (6), the stigma surface structure and colour $(7,8)$, the diameter of the flower (9) vertical/erect sepals (10), the colour of the underside leafblade (11), the nervation of the leafblade (12), the direction of the main nerves leafslips (13) and the proportion of the stigma to the width of the ovary (14) and for some specimens also the pollen diameter and pollen exine morphology (values given in the distribution list). All collected individuals were determined in the field with careful measurement of the chosen features. Altogether, 64 flowers, 70 leaves and 15 ovaries belonging to 62 specimens were collected and checked. The average stigma diameters, the number of carpellary teeth and the flower diameter were compared to the values of $N$. alba. Pollen diameter was measured using the Olympus BX41 microscope with objective UPlan SA $100 \times 00 / 017 /$ FN 26.5 and photographed by the Olympus Camedia camera C3040 200M. Altogether pollens from 73 populations of $N$. candida and 18 of $N$. alba from all the researched areas were measured. Half of them was sampled from fresh material, another half from herbarium collections (WRSL, KRA). The collected material was stored in the herbarium of the Division of Geobotany and Plant Conservation of the Opole University (OPUN).

The trophic level of an ecosystem is evaluated according to the results of the total nitrogen, total phosphorus, chlorophyll $a$, water transparency and biological parameters (Dodds et al. 1998). However, it is generally admitted that phosphorus plays an important role in the development of aquatic plants and, in most cases, is a limiting factor of eutrophication in temperate lakes (Parinet et al. 2004). In order to estimate the status of investigated water bodies, the Trophic State Index (TSI) was calculated according to Carlson's equations (Carlson 1977):

$$
\mathrm{TSI}=\frac{T S I(T P)+T S I(C h l)+T S I(S D)}{3}
$$

where:

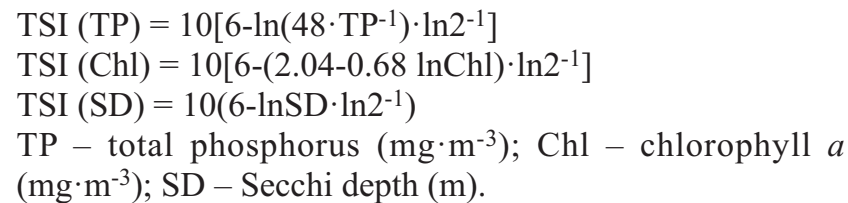

TSI $(\mathrm{Chl})=10\left[6-(2.04-0.68 \operatorname{lnChl}) \cdot \ln 2^{-1}\right]$

TSI $(\mathrm{SD})=10\left(6-\operatorname{lnSD} \cdot \ln 2^{-1}\right)$

$\left(\mathrm{mg} \cdot \mathrm{m}^{-3}\right) ; \mathrm{SD}-$ Secchi depth $(\mathrm{m})$

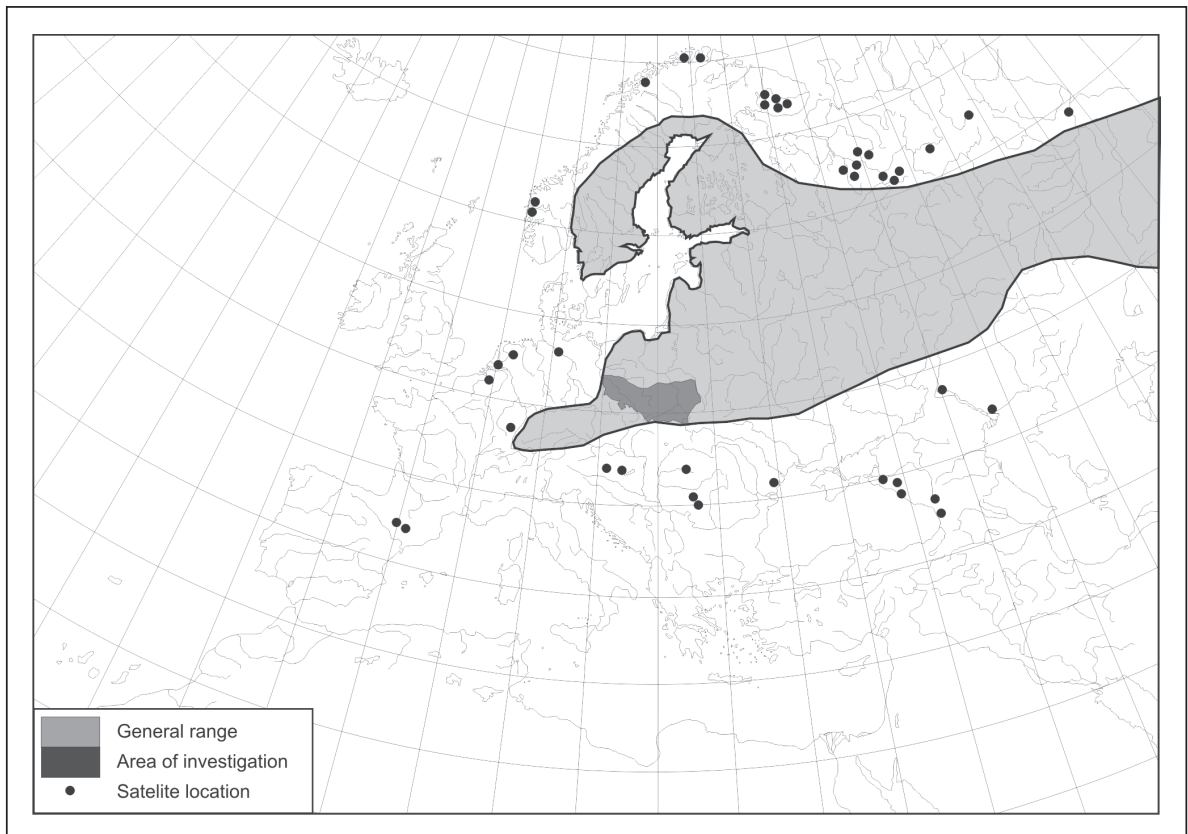

Fig. 1. The distribution range of Nymphaea candida in Europe with the investigation area. 


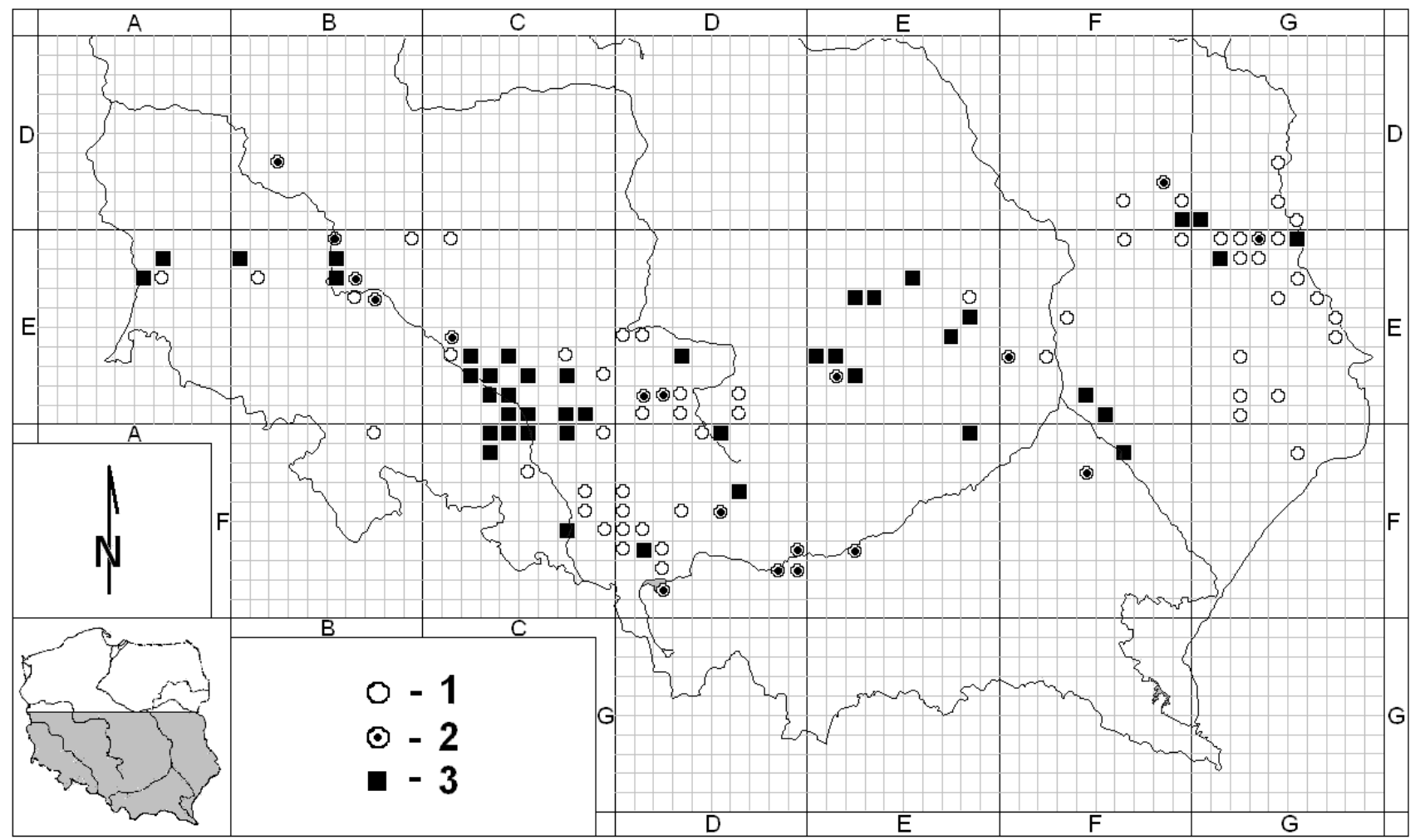

Fig. 2. Distribution of Nymphaea candida in southern Poland on the background of ATPOL grid. 1 - locations known before the year $2001 ; 2-$ locations known before the year 2001, documented with herbarium specimens verified according to methods described in the paper; 3 - locations noted after 2001.

The TSI values, constructed on a scale of 0 to 100 , indicate the trophic level of ecosystems from oligotrophic $(<40)$ to eutrophic (60-100). To minimize the sampling mistake the mean value of three indices $\left(\mathrm{TSI}_{\mathrm{TP}}, \mathrm{TSI}_{\mathrm{Chl}}\right.$ and $\mathrm{TSI}_{\mathrm{SD}}$ ) were taken while determining the trophy level (Pełechaty et al. 2007). Also the reference classification adopted by OECD was taken into account (OECD 1982; Solheim 2005).

The endanger status of $N$. candida in southern Poland was evaluated according to the IUCN categorization method (IUCN 2001). All doubtful specimens, probably hybrids, were excluded from the analyses. Only evident individuals of Nymphaea candida were taken into account when generating map and listing the location of the species.

The average values of the pollen diameter of both species were compared using the $t$ test for independent variables.

\section{RESULTS}

THE DISTRIBUTION

OF NYMPHAEA CANDIDA IN SOUTHERN POLAND

All localities of $N$. candida from southern Poland published before 2001 are listed below. Additionally, new records as well as old localities which have been confirmed recently (after 2001) in the field are also presented (Fig. 2).

\section{Abbreviations (relate to Results):}

$\mathrm{GB}$ - dense exine outgrowths; $\mathrm{RB}$ - rare exine outgrowths

By location the position in ATPOL grid $10 \times 10 \mathrm{~km}$ (Zając 1978) was given. If the precise localisation of a population in ATPOL grid was imposible, a question-mark (?) was given.

\section{Lower Silesian Province}

AE16: Jagodzin (Barber 1937; Schube 1903a), Węgliniec - Dziczy Pond (Schube 1903a), Węgliniec - Wolno Stary Pond (Schube 1903a), Węgliniec - Wolno Nowy Pond (Berdowski and Narkiewicz 1996);

AE25: Zielonka - pond to the NE from the village (Zabawski and Matuła 1973), Zielonka - Krusza Pond (Berdowski and Narkiewicz 1996), Zielonka - Rygle I Pond - western reservoir (Berdowski and Narkiewicz 1996), Zielonka - Rygle I Pond - eastern reservoir (Berdowski and Narkiewicz 1996), Zielonka - bog to the NE from Rygle I Pond (Berdowski and Narkiewicz 1996), Zielonka - Rygle II (Berdowski and Narkiewicz 1996), Zielonka midforest pond to the SE from Rygle II Pond - forest division section 201 (Berdowski and Narkiewicz 1996);

AE26: Zielonka - pond to the NE from the village (Zabawski and Matuła 1973), Zielonka - pond close to the railway, to the NE from Zielonka (Berdowski and Narkiewicz 1996), Węgliniec - bog to the NW from railway station (Schube 1903a; Zabawski and Matuła 1973);

BD62: Radzyń near Sława (leg. E. Kozioł 04.07.1974, WRSL 94073, pollen ø 42-45 $\mu \mathrm{m}, \mathrm{GB})$;

BE05: Przychowa near Legnica, Odra river ox-bow lake (leg. E. Kozioł 28.06.1993, WRSL 87085, pollen ø (40)42-43 $\mu \mathrm{m}, \mathrm{GB}$ ); Przyborów, Odra river ox-bow lake (Macicka and Wilczyńska 1992; Macicka-Pawlik and Wilczyńska 1996, 1998; Bobrowicz and Konieczny 2000);

BE09: Niezgoda (Schube 1903a), Ruda Sułowska (Schube 1903a), Radziądz (Anioł-Kwiatkowska et al. 1995);

BE10: Nature Reserve "Torfowisko Borówki” (Cieślak and Szlachetka 1987; Szlachetka and Szlachetka 1998), "Sześć Stawów" (Cieślak and Szlachetka 1987);

BE15: Tarchalice - Odra river ox-bow lake (MacickaPawlik and Wilczyńska 1996, 1998), Tarchalice, ox-bow lake (leg. T. Macicka 12.07.1991, WRSL 82980, pollen ø 


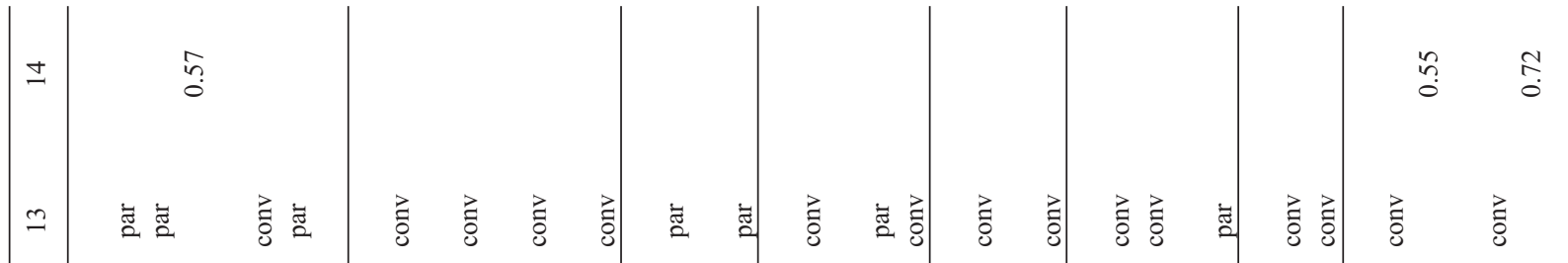

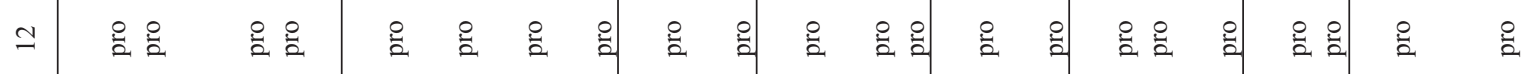

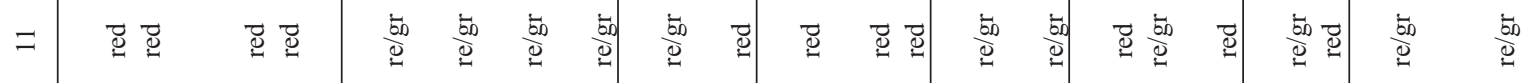

○

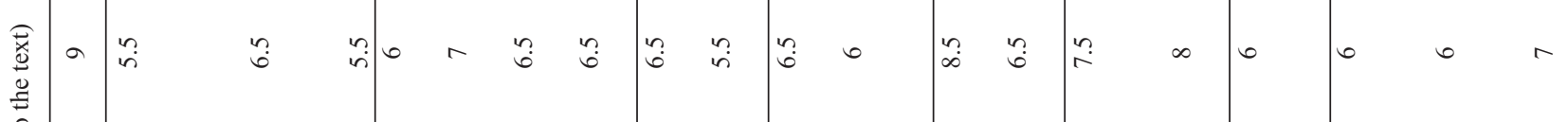

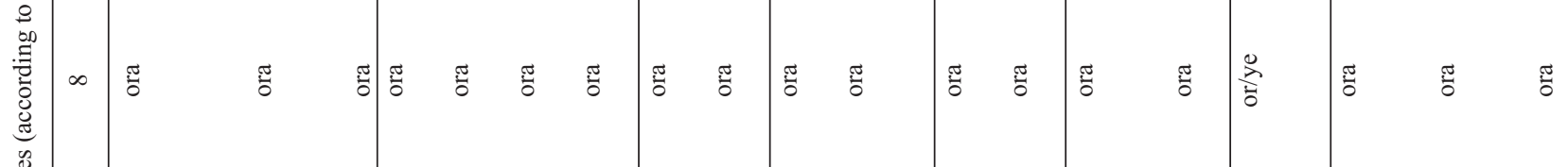

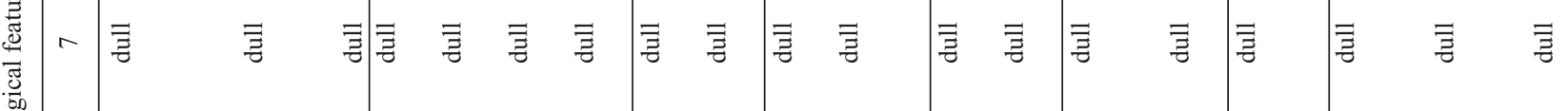

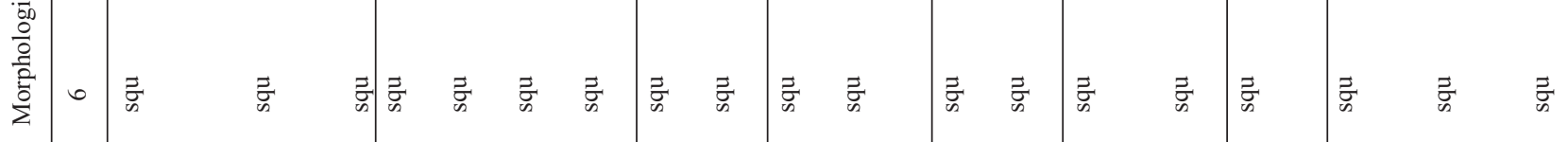

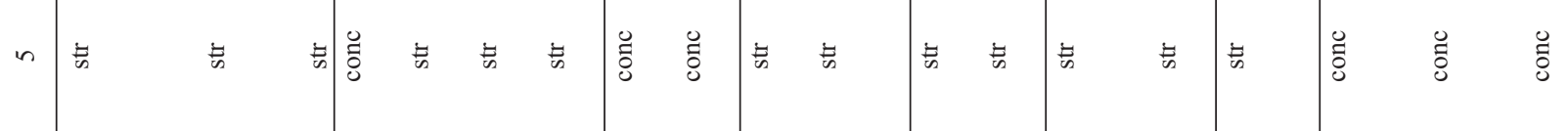

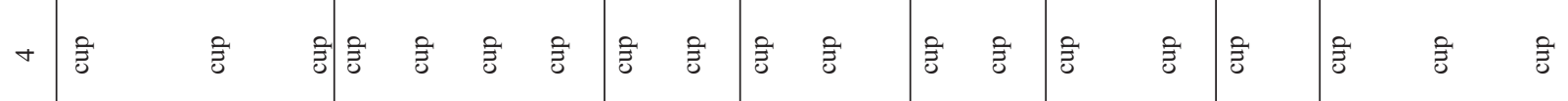

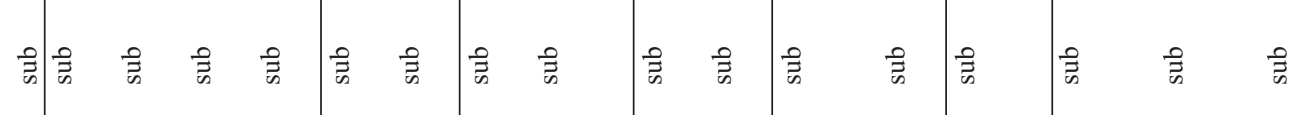

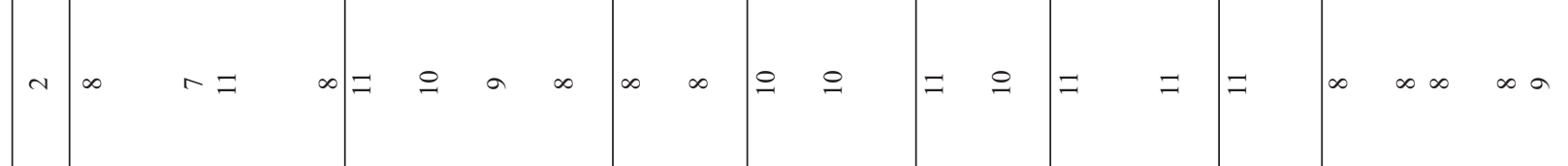

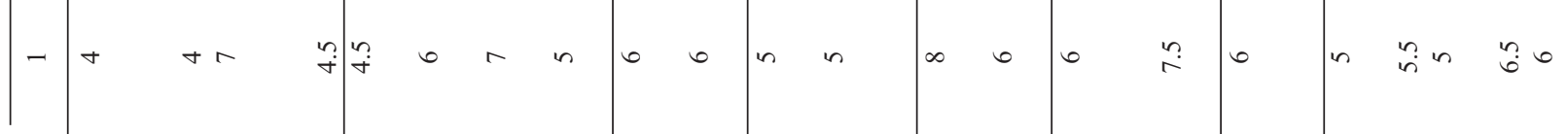

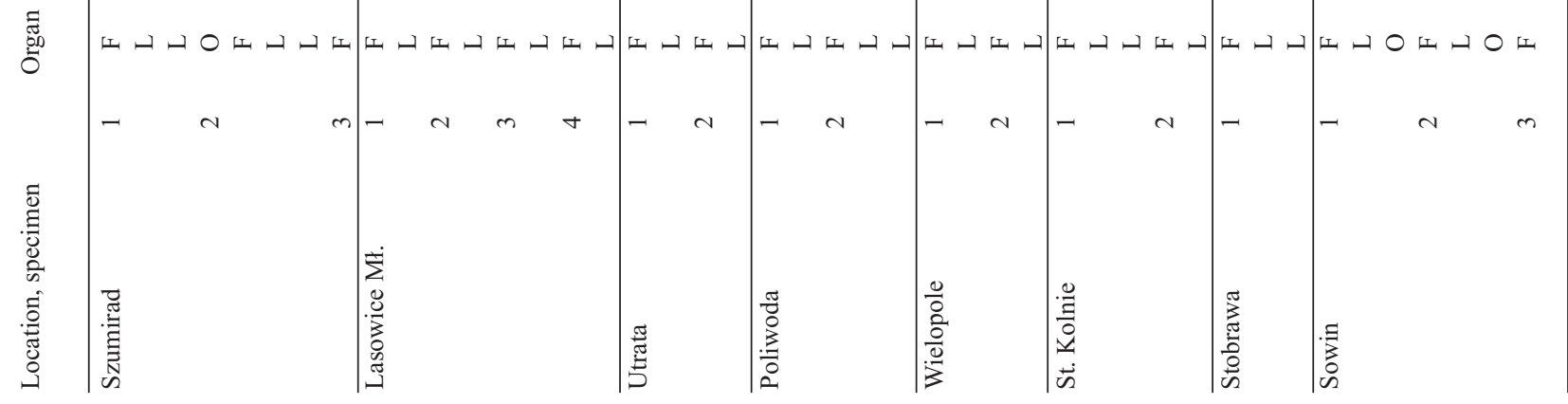


338

NYMPHAEA CANDIDA IN SOUTH POLAND

Nowak A. et al.

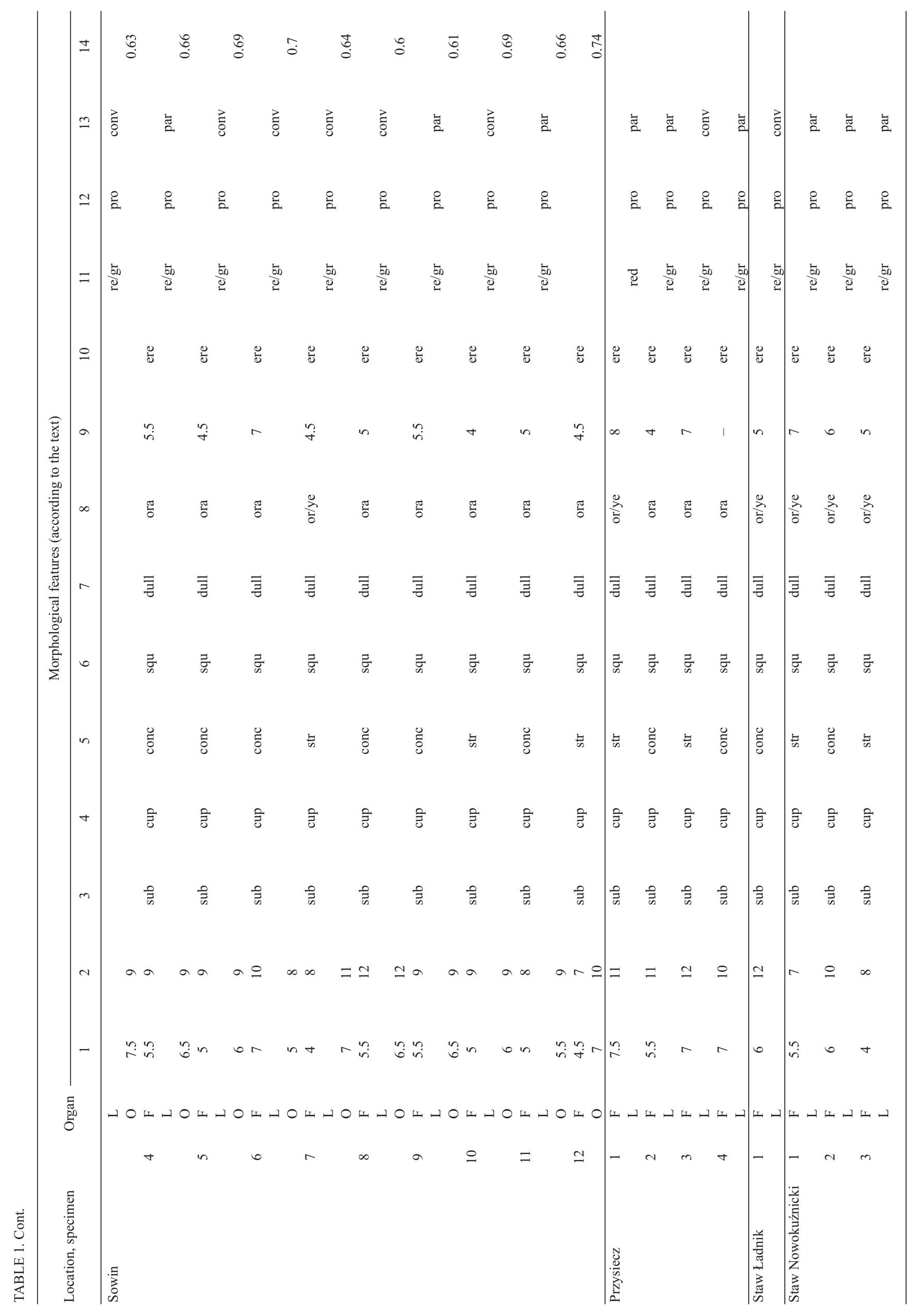




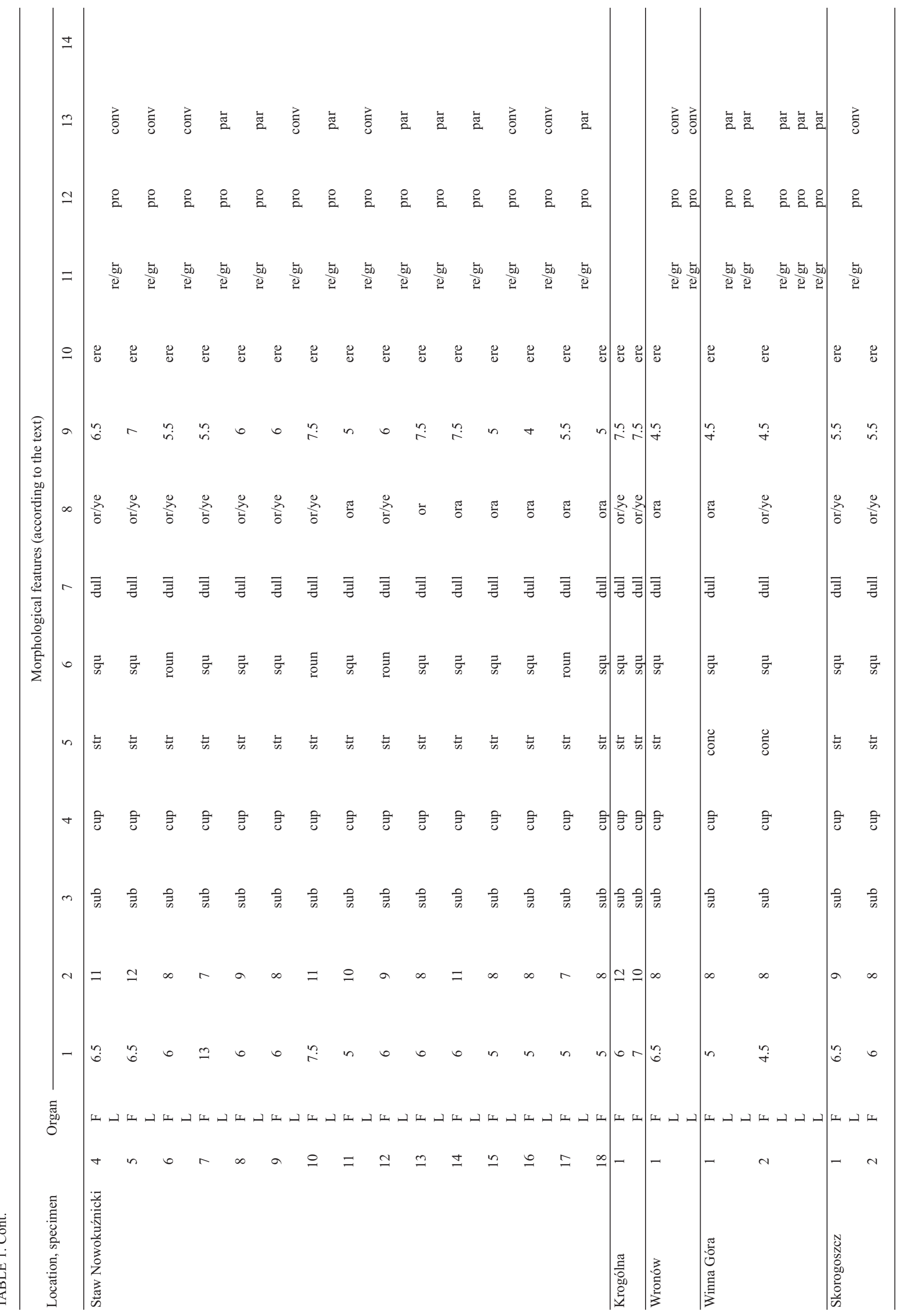




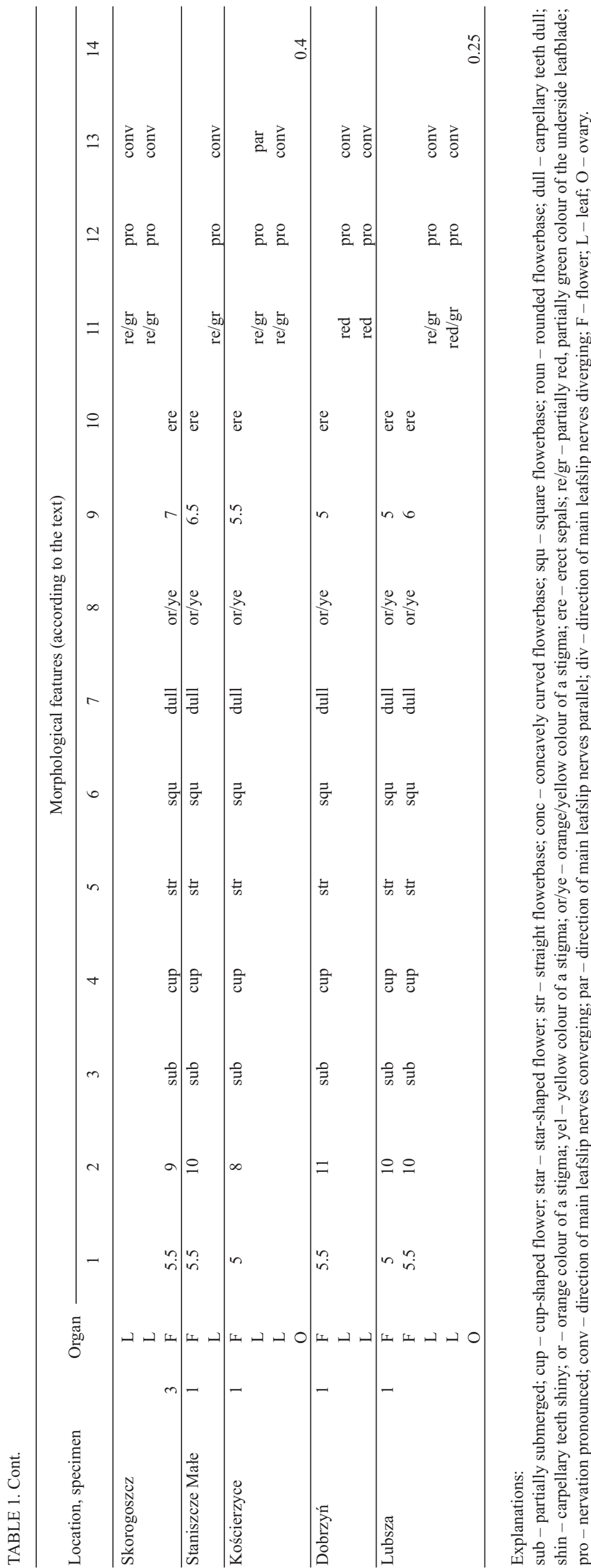

42.5-45 $\mu \mathrm{m}, \mathrm{GB})$, Tarchalice, ox-bow lake (leg. T. Macicka 23.07.1991, WRSL 82863, pollen ø 37.5-45 $\mu \mathrm{m}, \mathrm{GB})$, Boraszyn - Odra river ox-bow lake (Macicka-Pawlik and Wilczyńska 1996, 1998), Boraszyn near Wrocław, ox-bow lake (leg. T. Macicka 12.07.1991, WRSL 82979, pollen ø 37.5-45 $\mu \mathrm{m}, \mathrm{GB})$; Dziewin-Grzybów; Odra river ox-bow lake (leg. E. Kozioł 29.06.1993, WRSL 87086, pollen ø $43 \mu \mathrm{m}, \mathrm{GB})$; Dziewin by Ścinawa, ox-bow lake (leg. E. Kozioł 04.07.1993, WRSL 87095, pollen ø 42-43 $\mu \mathrm{m}, \mathrm{GB})$;

BE21: Jaroszówka near Chojnów (Schube 1903a);

BE25: Jurcz near Ścinawa, Odra river ox-bow lake (1 specimen leg. E. Kozioł 29.06.1993, WRSL 87087, pollen ø 41-43 $\mu \mathrm{m}$, GB; 2 specimens leg. E. Kozioł 15.07.1993, WRSL 87088, pollen $\varnothing$ 40-41 $\mu \mathrm{m}, \mathrm{GB} ; 3$ specimens leg. E. Kozioł 15.07.1993, WRSL 87089, pollen ø 41-45 $\mu \mathrm{m}, \mathrm{GB})$; Lubiąż, Nature Reserve "Odrzyska" (leg. T. Macicka 26.07.1991, WRSL 82951, pollen ø 40-46 $\mu \mathrm{m}, \mathrm{GB})$;

BE26: Wołów, clay pit (leg. Z. Głowacki 14.07.1964, WRSL 71967, pollen ø 43.75-46.25 $\mu \mathrm{m}, \mathrm{GB})$;

BE36: Grodzanów near Wołów (Schube 1925), Słup - Odra river ox-bow lake (Macicka-Pawlik and Wilczyńska 1996, 1998);

BE37: Szczepanów near Środa Śląska, forest pond (leg. A. Krawiecowa 04.07.1963, WRSL 66471, pollen ø 43-47 $\mu \mathrm{m}, \mathrm{GB})$;

BF07: Ząbkowice Śląskie (Schube 1904);

CE01: Grabownica near Milicz (Schube 1903a);

CE51: Jelcz, Odra river ox-bow lake (leg. E. Krawiecówna 06.07.1960; WRSL 34396, pollen ø 43-45 $\mu \mathrm{m}, \mathrm{GB})$;

CE61: Stary Otok (Anioł-Kwiatkowska et al. 1998).

Misleading locations: Klosterteich pond by Lubomierz (Schallow 1935) - according to the author comment it was an anthropogenic population.

\section{Lublin Province}

FD78: Mizak Lake, 04.07.1964, rev. M. Kucharczyk 1995; pollen ø 40-45 $\mu \mathrm{m}$, GB; Fijałkowski 1962); Świerże, Tyśmienica river (Fijałkowski 1962);

FD86: Giżyce by Kock, Wieprz river (Fijałkowski 1964);

FD78: Skoki by Czemiernik, Tyśmienica river (Fijałkowski 1964);

FD89: Siemień, fish ponds (Fijałkowski 1962);

FD99: Kleszczów Lake, Łęczyńsko-Włodawskie Lakeland (Fijałkowski 1959a; Popiołek 1971; Sugier and Czarnecka 1998); Czarne Gościnieckie Lake (Fijałkowski 1959a; Popiołek 1974; Sugier 1998); peat-bog depressions by Miejskie Lake (Popiołek 1973; Czarnecka and Sugier 1998);

FE06: Samoklęski, fish ponds (Fijałkowski 1962); 
FE09: Rudka Kijańska, ditches (Fijałkowski 1962);

FE43: Jezioro Bartków (Fijałkowski 1961);

FE62: Popów, Bliskowice, Świeciechów Duży, Wisła river (Fijałkowski 1960);

FE84: Zaklików, Stawy (Fijałkowski 1960);

FE95: Nature Reserve "Stawy Wilczowskie" (Fijałkowski 1997); Nature Reserve "Imielty Ług" (Fijałkowski et al. 1992; Fijałkowski 1997); Nature Reserve "Lasy Janowskie" (Fijałkowski 1997);

GD64: Holeszów by Sławatycze, meadow ditch (Fijałkowski 1963);

GD84: Dołhobrody by Włodawa, Bug river (Fijałkowski 1963);

GD95: Surroundings of Sobibor by Włodawa, Bug river (Fijałkowski 1964);

GD90: Skomielno Lake, Łęczyńsko-Włodawskie Lakeland (leg. D. Fijałkowski 01.08.1957, pollen ø $48 \mu \mathrm{m}$, GB; Fijałkowski 1959a);

GE01: Karaśne Lake by Wujek (Fijałkowski 1959a; Sugier, Popiołek 1995); Moszne Lake (Fijałkowski 1959a; Sugier and Popiołek 1998);

GE02: Lubowierz Lake, Lubowierzek Lake (Fijałkowski 1959a);

GE03: Dubeczyńskie Lake, Łęczyńsko-Włodawskie Lakeland (leg. D. Fijałkowski 14.08.1958; pollen ø (36)43$50 \mu \mathrm{m}, \mathrm{GB}$; Fijałkowski 1959a; Fijałkowski et al. 1994a); Hańskie Lake, Pojezierze Łęczyńsko-Włodawskie (leg. D. Fijałkowski 26.08.1960, pollen ø 40-41 $\mu \mathrm{m}$, R-GB; Fijałkowski 1959a);

GE04: Spilno Lake (Fijałkowski et al. 1994a); Perespilno Lake (Fijałkowski 1959a; Fijałkowski et al. 1994b);

GE05: Płotycze Lake, Sobiborskie Forests, ŁęczyńskoWłodawskie Lakeland (Fijałkowski 1959a; Fijałkowski et al. 1993); Brudno Lake, Sobiborskie Forest, ŁęczyńskoWłodawskie Lakeland (Fijałkowski 1959a; Fijałkowski et al. 1993);

GE11: Eastern shore of Uściwierz Lake, Grabniak by Łęczna, Łęczyńsko-Włodawskie Lakeland (Fijałkowski 1959a; Popiołek 1988); Rotcze Lake (Fijałkowski 1959a; Popiołek 1988), Sumin Lake (Fijałkowski 1959a; Popiołek 1988); Bikcze Lake (Fijałkowski 1959a; Popiołek 1988); Nadrybie Lake (Fijałkowski 1959a; Popiołek 1988);

GE12: Świerszczów Lake (Fijałkowski 1959a, 1965; Karczmarz and Malicki 1971);

GE13: Słone Lake (Fijałkowski 1959a);

GE25: Hniszów, in Bug river (Fijałkowski 1964);

GE34: Kol. Rudka, Kamień, Strupie, peat-bog depressions in calcareous moors (Fijałkowski 1959a);

GE36: Dorohusk by Chełm, Bug river (Fijałkowski 1964);

GE47: Uchańka by Dubienka, Bug river (Fijałkowski 1964);

GE57: Matcze by Horodła, Bug river (Fijałkowski 1964);

GE62: Stryjów, Orłów Murowany, ponds (Fijałkowski 1960);

GE82: Sitaniec, Łabuńka river (Fijałkowski 1959b);

GE84: Miączyn, peat-bog depressions (Fijałkowski 1959b);

GE92: Lipsko, peat-bog depressions (Fijałkowski 1959b);

GF15: Chodywańce by Tomaszów Lubelski, peat-bog depressions (Fijałkowski 1962).

\section{Małopolska Province}

DF69: Kraków - Dębniki, data ?, leg. ?; KRA 11471; pollen q $42-46 \mu \mathrm{m}, \mathrm{GB})$;

DF78: Tyniec near Kraków - pond, leg. H. Błaszczyk may 1938, KRA 89674; ø pyłku 41-43 $\mu \mathrm{m}$ GB);

EF62: Puszcza Niepołomicka, Ispina, ox-bow lake "Wiślisko Kobyle" in Myriophyllo-Nupharetum association, leg. E. Dubiel 5.08.1968, KRA 98181; pollen ø 42-45 $\mu \mathrm{m} \mathrm{GB}$ );

DF69: Kraków - Dębniki, leg. F. Berdau 1854, KRA 11470; pollen ø 42,5-47,5 $\mu \mathrm{m}, \mathrm{GB})$;

DF79: Kraków - Kobierzyn, leg. F. Berdau 1854, KRA 11470; pollen ø 42,5-47,5, $\mu \mathrm{m}$ GB).

\section{Mazowsze Province}

EE38: Pakosławskie bog (Młyn Bagno) in Pakosław near Iłża (Szafran 1925);

FE60: Puszcza Kozienicka, bog near Anielin; leg. H.

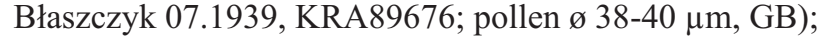

?: Puszcza Kozienicka, bog near Laskowola, drainage ditch (leg. H. Błaszczyk 6.07.1939, KRA 89677; pollen ø $42 \mu \mathrm{m}, \mathrm{GB})$.

\section{Opole Province}

CE67: Kluczbork (Fiek 1887; Schube 1903a);

CE73: Nowe Kolnie (Kuźniewski 1964);

CE77: Szumirad near Olesno, pond (Fiek 1888; Schube 1903a);

CE79: Borki Wielkie (Schube 1903b);

CE83: Buszyce near Lewin Brzeski (Schube 1908);

CE84: Chrócice (Wimmer 1844; Schube 1903a);

CE85: Kup (Schube 1903a);

CE98: Mid forest pond in Staniszcze Małe (Dajdok et al. 1998);

CF03: Tułowice Małe, Grodziec (Wimmer 1844; Schube 1903a), Tułowice (Schube 1910);

CF04: Przysiecz (Fiek, Schube 1893; Schube 1903a);

CF05: Staw Nowokuźnicki (Michalak 1963);

CF09: Zawadzkie (Dajdok et al. 1998);

CF13: Stara Jamka (Schube 1910);

CF25: Dobra (Schube 1906);

CF48: Stara Kuźnia (Wimmer 1844; Schube 1903a);

DE50: Dalachów, pond in forest sect. No. 96 (Fojcik 1997).

Misleading locations: Lewin Brzeski (Kuczyńska 1974 data from before 1939) - data refers undoubtedly to Buszyce near Lewin Brzeski (Schube 1908) not to Lewin Brzeski itself; Chróścina (Kuczyńska 1974 - data from before 1939) - data refers to Chróścice (Schube 1903a); Korfantów (Kuczyńska 1974 - data from before 1939) data refers probably to Stara Jamka near Korfantów (Schube 1910);

\section{Podkarpacie Province}

FF24: Płaskowyż Kolbuszowski, Brzóza (S. Loster, E. U. Zając, E. Dubiel, A. Zając 17.06.1975, KRA 11735; pollen $ø 43-45 \mu \mathrm{m}, \mathrm{RB})$.

\section{Silesian Province}

CF38: Rudziniec (Schube 1903a);

CF57: Nature Reserve "Lężczok" (Fiek 1881; Sendek 1986); Grabowiec Pond in Nature Reserve "Łężczok" (leg. 
S. Zerger 10.07.1960, WRSL 30632, pollen ø 46-49 $\mu \mathrm{m}$, GB); Brzeziniak Pond in Nature Reserve "Łężczok" (leg. S. Zerger 18.07.1960, WRSL 30633, pollen ø 44-46 $\mu \mathrm{m}$, GB);

CF59: Stanice (Fiek 1881; Schube 1903a); Rybnik, ponds in the Ruda River valley (Uechtritz 1864b; Fiek 1881; Schube 1903a);

DE51: Stawy settlement near Parzymiechy (Hereźniak 1986);

DE 81: Brzoza Pond near Lubockie (ca. $10 \mathrm{~km}$ to the $\mathrm{N}$ from Lubliniec), leg. A. Czylok, G. Kłys 04, 08, 2000, KRA 257174; pollen ø 40-50 $\mu \mathrm{m}, \mathrm{GB}$ );

DE82: Blachownia, Blachownia Lake (Hereźniak and Samosiej 1990); Blachownia near Częstochowa, pond, leg. H. Błaszczyk 15.08.1948, KRA 11458; pollen ø 45-50 $\mu \mathrm{m}$, GB);

DE83: Gorzelnia in Blachownia commune (Hereźniak 1986);

DE86: Stawki in Przyrów commune (Hereźniak 1986); Knieja near Św. Anna (Hereźniak et al. 2001); Julianka (Hereźniak 1986);

DE91: Hadra (Uechtritz 1864a; Fiek 1881; Schube 1903a);

DE93: Kijas near Konopiska (Hereźniak 1986);

DE96: Bystrzanowice in Janów commune (Hereźniak 1993);

DF04: Stara Kuźnica (Denisiuk and Grynia 1969);

DF30: Czekanów by Pyskowice (Schube 1905);

DF40: Nieborowice (Fiek 1881; Schube 1903a);

DF43: Mysłowice (Sophienhütte) at present MysłowicePiasek (Schube 1903a; Schube 1904)

DF45: Sosnowiec-Cieśle (Sendek 1984); ponds near Biała Przemsza by Szczakowa (leg. J. Walas 06.1954, KRA 126558; pollen ø 41,5-42,5 $\mu \mathrm{m}, \mathrm{GB})$;

DF50: Rybnik-Kamień (Urbisz 1996);

DF51: near Bełk (Fiek and Schube 1893; Schube 1903a); Orzesze-Zawiść (Celiński et al. 1979);

DF60: Rybnik Gotartowice (Fiek 1881; Schube 1903a); Ligota Rybnicka, Żory (Urbisz 1996); Staw Garbocz, to the North from Żory (Urbisz and Urbisz 1998);

DF61: Żory-Kleszczówka, Suszec-Rudziczka (Urbisz and Urbisz 1998);

DF62: Tychy-Paprocany (Uechtritz 1885; Fiek 1881; Schube 1903a; Toma 1994);

DF72: Near Pszczyna (Fiek 1881; Schube 1903a);

DF82: Goczałkowice-Zdrój; fish pond (leg. T. Stryczek 07.08.1960, WRSL 72171, pollen ø 42-45 $\mu \mathrm{m}$, GB).

\section{Świętokrzyskie Province}

EF08: Lakes in carst sink, to the E from Golejów near Staszów (Ochyra 1979);

EE71: Out of use bog by Marianów and Mieczyn in Małogoskie Hills in Świętokrzyskie Mts (leg. K. Pstrokońska, T. Tacik, 6.07.1955; KRA11483; pollen ø $45 \mu \mathrm{m}$, GB);

?: Pasieki by Włoszczowa, Włoszczowski District pond in pine wood (leg. H. Błaszczyk, 6.07.1949; KRA 111973; pollen ø $45 \mu \mathrm{m}, \mathrm{GB})$;

?: Peat-bog near Włoszczowa (leg. H. Błaszczyk 29.08.1927, KRA 89675; pollen ø $45 \mu \mathrm{m}, \mathrm{GB})$.
The list of new localities found or confirmed by authors:

\section{Lower Silesian Province}

AE16: Stary Weggliniec, drainage ditch to the $\mathrm{W}$ from Wolno Stary Pond (W. Bena 2003 npbl, Z. Dajdok, Z. Kącki and K. Pender 2004 npbl., K. Pender and Z. Dajdok 2007 npbl); Węgliniec - Tartaczny Pond (obs. Z. Dajdok, A. Nowak, W. Bena 22.08.2009; pollen ø 40-44 $\mu \mathrm{m}, \mathrm{GB}$ );

AE25: Zielonka - bog to the NE from Rygle I Pond (W. Bena, Z. Dajdok, A. Nowak and J. Zalewska-Gałosz 2008 npbl), Zielonka - mid forest pond to the S from Rygle II (obs. W. Bena, Z. Dajdok, A. Nowak and J. ZalewskaGałosz 22.08.2009; pollen ø 44.5-46 $\mu \mathrm{m}, \mathrm{GB})$;

BE10: "Sześć Stawów" (obs. Z. Dajdok, A. Nowak, W. Bena 22.08.2009; pollen ø 42-45 $\mu \mathrm{m}$, GB), between Krzyżowa and Borówki - fish pond in forest (Szlachetka 2007a, 2008);

BE15: Małowice - large ox-bow lake to the $\mathrm{W}$ from Małowice (Ruszlewicz and Szlachetka 2007), Dziewin ox-bow lake to the S from village (Szlachetka 2007b);

BE25: Grzybów - ox-bow lake ca. $300 \mathrm{~m}$ to the SE from village (Szlachetka 2007b), Jurcz - ox-bow lake ca. 2500 $\mathrm{m}$ to the SW from village (Szlachetka 2007b), Jurcz - four small ox-bow lakes, ca. $1300 \mathrm{~m}$ to the $\mathrm{E}$ from village (Szlachetka 2007b), Jurcz - ca. $1200 \mathrm{~m}$ to the SE from village (A. Szlachetka 2007 npbl.), Kwiatkowice - ox-bow lake ca. $1200 \mathrm{~m}$ to the NE from village (Szlachetka 2007b).

\section{Lublin Province}

FD99: Kleszczów Lake, Łęczyńsko-Włodawskie Lakeland (obs. P. Sugier 04.09.2009; pollen ø $43 \mu \mathrm{m}, \mathrm{GB}$ );

GD90: Skomielno Lake near Orzechów, ŁęczyńskoWłodawskie Lakeland (obs. J. Zalewska-Gałosz 26.08.2002; KRA 207124; pollen ø $42 \mu \mathrm{m}, \mathrm{GB}$ );

GE05: Płotycze Lake, Sobiborskie Forest, ŁęczyńskoWłodawskie Lakeland (obs. P. Sugier 05.09.2009; 1 specimen pollen $\varnothing ~ 49-50 \mu \mathrm{m}, \mathrm{GB}, 2$ specimens, pollen $\varnothing ~ 44-46$ $\mu \mathrm{m}, \mathrm{GB}, 3$ specimens, pollen ø 43-45 $\mu \mathrm{m}, \mathrm{GB})$; Brudno Lake, Sobiborskie Forest, Łęczyńsko-Włodawskie Lakeland; 1 specimen, obs. P. Sugier 05.09.2009; pollen ø (35)46-49 $\mu \mathrm{m}, \mathrm{GB}$; 2 specimens, obs. P. Sugier 04.09.2009, pollen ø 45-51 $\mu \mathrm{m}, \mathrm{GB})$;

GE11: Uściwierz Lake, E part, Grabniak by Łęczna, Łęczyńsko-Włodawskie Lakeland, obs. 24.06.2004, J. Zalewska-Gałosz, KRA264290, 264261; pollen ø 40, 42, $45 \mu \mathrm{m}, \mathrm{GB})$.

\section{Małopolska Province}

DF36: Laski by Olkusz - small dam reservoirs on the Biała river (leg. W. Bartoszek, M. Radoszek, J. Żelazny, 11.06.2008, KRA 35764; pollen ø $50 \mu \mathrm{m}, \mathrm{GB})$.

\section{Mazowsze Province}

EE48: Iłża, Błaziny Dolne and Budki Seredzkie, reservoir on the Iłżanka river. Pond in Kolonia Seredzice (Nobis 2007; M. Nobis 2008 npbl.); dam reservoir on the Iłżanka river by Błaziny Dolne near Iłża - to the S from Radom (obs. M. Nobis 09.09.2003, KRA 265617; pollen ø 42, 45 $\mu \mathrm{m}, \mathrm{GB}$ ); Iłża, dam reservoir (obs. M. Nobis 16.06.2003, KRA 357645; pollen ø 37,5-42, GB).

\section{Opole Province}

CE62: Ox-bow lake in Błota (obs. A. Nowak 16.07.2008); 
CE64: Krogólna, fish ponds (Nowak and Nowak 2007; leg. A. Nowak 08.2007; pollen ø 35-43 $\mu \mathrm{m}, \mathrm{GB}$ );

CE72: Ox-bow to the S from Lubsza and to the SW and NE from Kościerzyce (obs. A. Nowak 14.07.2008);

CE73: Odra river ox-bow lake in Stare Kolnie (Nowak and Nowak 2007; leg. A. Nowak 08.2007, pollen ø 43-46 $\mu \mathrm{m}, \mathrm{GB}$ ); Odra river ox-bow lake in Stobrawa (Nowak and Nowak 2007, leg. A. Nowak 08.2007; pollen ø 44-49 $\mu \mathrm{m}$, GB);

CE75: Winna Góra, fish ponds (Nowak and Nowak 2007, leg. A. Nowak 08.08.2007; pollen ø of 2 specimens, each $46 \mu \mathrm{m}, \mathrm{GB})$;

CE77: Szumirad near Olesno, pond (Nowak and Nowak 2007; leg. A. Nowak 08.2007; 1 specimen pollen ø 43-46 $\mu \mathrm{m}, \mathrm{GB} ; 2$ specimens pollen $\varnothing 48-49 \mu \mathrm{m}, \mathrm{GB} ; 3$ specimens pollen ø 46-50 $\mu \mathrm{m}, \mathrm{GB})$; between Lasowice Małe and Lasowice Wielkie, pond (Nowak and Nowak 2007); Lasowice Małe - village pond, leg. A. Nowak 08.2007, 1 specimen KRA 358250; pollen ø 46-48 $\mu \mathrm{m}$, GB; 2 specimens KRA 358006, pollen ø (44)45-48 $\mu \mathrm{m}, \mathrm{GB})$;

CE83: Odra river ox-bow lake in Wronów (leg. A. Nowak 09.08.2007; pollen ø 42-43 $\mu \mathrm{m}$, GB) Nysa Kłodzka ox-bow lake in Skorogoszcz (Nowak and Nowak 2007; leg. A. Nowak 08.08.2007; pollen ø $43 \mu \mathrm{m}, \mathrm{GB})$;

CE84: Wielopole by Popielów, Odra river ox-bow lake (Nowak and Nowak 2007; leg. A. Nowak 12.08.2007; pollen $\varnothing 41 \mu \mathrm{m}, \mathrm{GB})$, Odra river ox-bow lake to the $\mathrm{N}$ from Narok and in Kopanie (obs. A. Nowak 14.07.2008);

CE94: Odra river ox-bow lake between Niewodniki and Żelazna (obs. A. Nowak 14.07.2008);

CE95: Odra river ox-bow lake in Żelazna (obs. A. Nowak 14.07.2008);

CE97: Poliwoda, ponds (Nowak and Nowak 2007);

CE98: Mid forest pond in Staniszcze Małe (Nowak and Nowak 2007; leg. A. Nowak 08.2007; pollen ø 43-46 $\mu \mathrm{m}$, GB);

CF03: Pustelnik and Loża ponds by Lipno, Lawnik pond by Tułowice (Nowak and Nowak 2007);

CF04: Przysiecz (Nowak and Nowak 2007; leg. A. Nowak 23.08.2007; 1 specimen pollen ø 40-43 $\mu \mathrm{m}, \mathrm{GB} ; 2$ specimens, pollen ø 40-43 $\mu \mathrm{m}, \mathrm{GB})$;

CF05: Nature Reserve "Staw Nowokuźnicki" (Nowak and Nowak 2007; leg. A. Nowak 08.2007, 1 specimen pollen $\varnothing 46 \mu \mathrm{m}, \mathrm{GB} ; 2$ specimens pollen $\varnothing 40-43 \mu \mathrm{m}, \mathrm{GB} ; 3$ specimens pollen $\varnothing(38) 40-43$; $\mu \mathrm{m}, \mathrm{GB})$; Opole-Groszowice, pond (Nowak and Nowak 2007);

CF07: Utrata, pond (Nowak and Nowak 2007);

CF13: Mid forest pond to the $\mathrm{S}$ from Sowin (leg. A. Nowak 08.2007, Nowak and Nowak 2007; 1 specimen pollen $\varnothing ~ 43-46 \mu \mathrm{m}, \mathrm{GB} ; 2$ specimens pollen $\varnothing 46-48 \mu \mathrm{m}$, GB).

\section{Podkarpacie Province}

FF16: Pond in Tanew river valley, to the $\mathrm{N}$ from Nadtamy settlements by Ulanów (A. Nobis 2005 npbl);

FE84: Jan Pond by Zaklików in Sandomierska Basin (leg. R. Krawczyk, 07.08.2003, KRA 357977; pollen ø 42$45 \mu \mathrm{m}, \mathrm{GB})$;

FE95: Świdry in Sandomierska Basin, Oleńka pond (leg. R. Krawczyk 07.08.2003, KRA 357638; ø of pollen, 42-43 $\mu \mathrm{m}, \mathrm{GB})$.

\section{Silesian Province}

CF57: Nature Reserve "Łężczok" (A. Stebel 2008 npbl);

DF05: Myszków-Osińska Góra (Fojcik 2003);

DE63: Kocin Nowy in Mykanów commune (R. Bula and K. Henel $2006 \mathrm{npbl}$ );

DF61: Woszczyce, Widek pond (A. Stebel 2008 npbl).

\section{Świętokrzyskie Province}

EE25: Mid forest lake on Jabłonica river in Rzuców near Chlewiska (obs. M. Nobis 26.08.2003, KRA 265084; pollen ø 42-45 $\mu \mathrm{m}, \mathrm{GB})$; Rzuców - mesotrophic pond (obs. M. Nobis 26.08.2003, KRA 357971; pollen ø 40-43 $\mu \mathrm{m}, \mathrm{GB})$;

EE32: Mesotrophic pond by Barycz near Końskie (A. Trojecka $2007 \mathrm{npbl)}$;

EE33: Mesotrophic pond by Stary Młyn near Końskie (A. Trojecka 2007 npbl);

EE57: Pasternik reservoir on Kamienna river in Starachowice (obs. M. Nobis 08.09.2003, KRA 265037; Nobis 2007; M. Nobis 2008 npbl.; pollen ø 45, $50 \mu \mathrm{m}, \mathrm{GB})$; Pasternik reservoir on Kamienna river in Starachowice (obs. M. Nobis 08.09.2003, KRA 265085; pollen ø 40, 43, 45, $48 \mu \mathrm{m}, \mathrm{GB})$; Pasternik reservoir on Kamienna river in Starachowice (obs. M. Nobis 08.09.2003, KRA 265619, 265622; pollen ø 42, $45 \mu \mathrm{m}, \mathrm{GB})$;

EE60: Peat-bog pond near Skąpe village (Łopuszańskie Hills), (obs. A. Adamiec, R. Piwowarczyk 12.08.2008);

EE61: Peat-bog pond near Mokre village (Łopuszańskie Hills; obs. A. Adamiec 12.08.2008 npbl);

EE72: Lake within transitional bogs "Żarnowski Ług" by Gnieździska (Łopuszańskie Hills; obs. 14.08.2008 A. Adamiec, R. Piwowarczyk npbl);

EF08: Lakes in carst sink, to the E from Golejów near Staszów (M. Nobis 2006, $2008 \mathrm{npbl}$ ).

\section{MORPHOLOGICAL FEATURES OF THE COLLECTED SPECIMENS}

The field and laboratory measurements of the collected 62 specimens are presented in Table 1. Altogether 64 flowers, 70 leaves and 15 ovaries were collected and determined. The three most diagnostic characters were compared in Table 2 with values known for Nymphaea alba (Nowak and Nowak 2007).

The mean value of the stigma diameter for the collected flowers and ovaries is ca. $5.87 \mathrm{~mm}$ ranging from 4 to 13 (Table 2, Fig. 3). The average number of carpellary teeth meets perfectly the strict $N$. candida range of this features and counts ca. 9.29, ranging from 7 to 12 (Table 2, Fig. 4). Also the flowers of the verified specimens were small with the diameter restricted to the $N$. candida profile. The average value of this characteristic was $5.95 \mathrm{~cm}$ ranging from 4 to 8.5 (Table 2, Fig. 5).

The pollen diameter was measured for 98 specimens representing 73 populations of $N$. candida and for 31 specimens representing 18 populations of $N$. alba. The average value of the parameter was for $N$. candida $43.95 \mu \mathrm{m}$ and for N. alba $33.63 \mu \mathrm{m}$ (Table 2, Figs 6-8). The pollen diameter for both species has statistically different values $(\mathrm{p}<0.001$; Fig. 8). 
TABLE 2. Mean values of quantitative features for Nymphaea candida and N. alba.

\begin{tabular}{|c|c|c|c|c|}
\hline & Stigma diameter $[\mathrm{mm}]$ & Number of carpellary teeth & Flower diameter $[\mathrm{cm}]$ & Pollen diameter $[\mu \mathrm{m}]$ \\
\hline N. candida & 5.87 & 9.29 & 5.95 & 43.95 \\
\hline N. alba & $13.4^{*}$ & $16.1^{*}$ & $11.2 *$ & 33.63 \\
\hline
\end{tabular}

* according to Nowak and Nowak (2007)

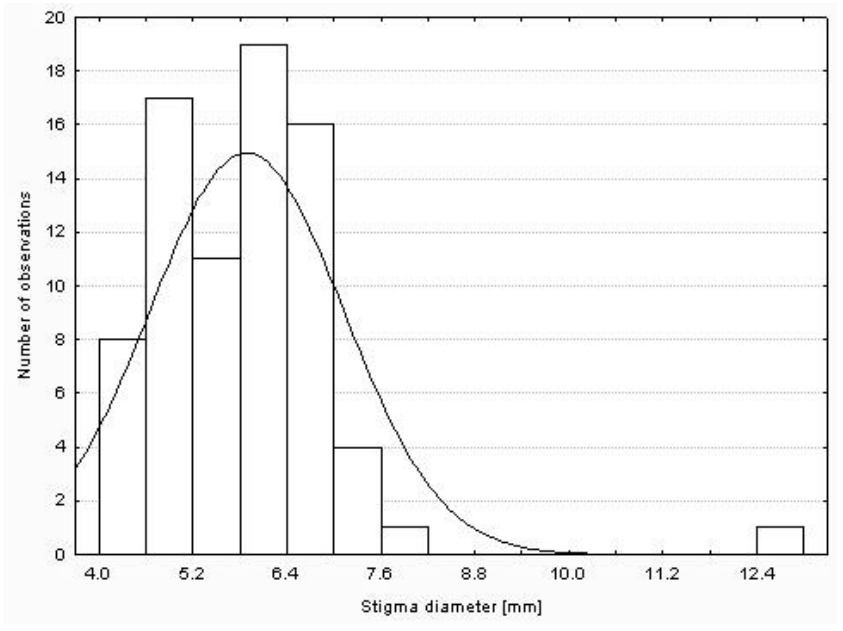

Fig. 3. Values of stigma diameter of $N$. candida specimens.

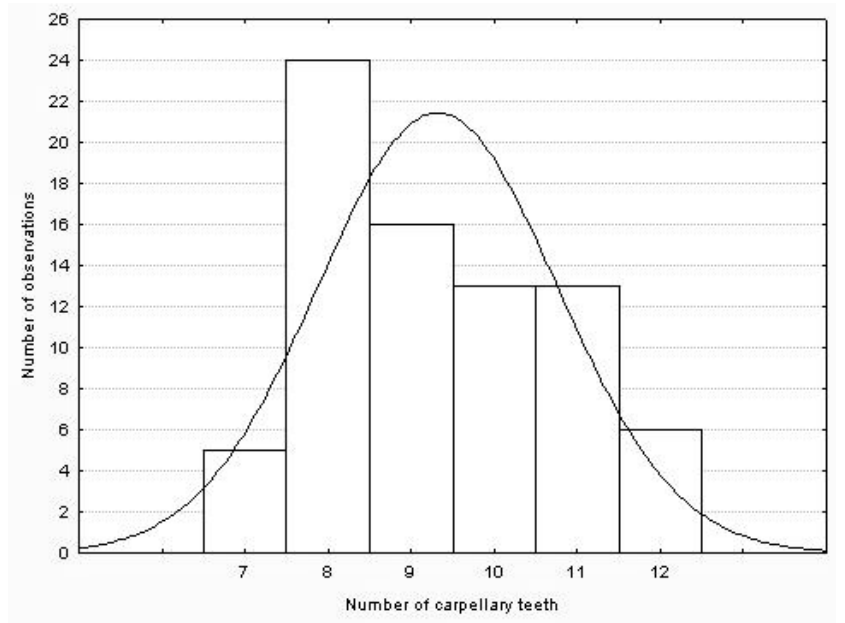

Fig. 4. Number of carpellary teeth of $N$. candida specimens.

\section{HABITAT PREFERENCES}

\section{OF NyMPHAEA CANDIDA C. PRESL IN SOUTHERN POLAND}

The populations of $N$. candida in southern Poland occupy mainly water bodies especially surrounded by forests, but in some cases also river ox-bow lakes within the meadow-field landscapes. The most suitable habitat conditions for this species occur in shallow waters in the shore zone with the amplitude of the water column fluctuating from 0.5 to $2 \mathrm{~m}$. Populations of $N$. candida develop on open water or close to the rushes with high insolation rate. Higher shading influences directly on the decrease of density and cover rate of the species. In thick reeds or high rushes the species occurs sporadically with a low population size.

The significant number of these populations were found in artificial reservoirs - fish ponds or forest ponds. Regarding the trophy level $N$. candida occupies different habitats, mainly mesotrophic but also eutrophic with high content of organic matters (Table 3 ).

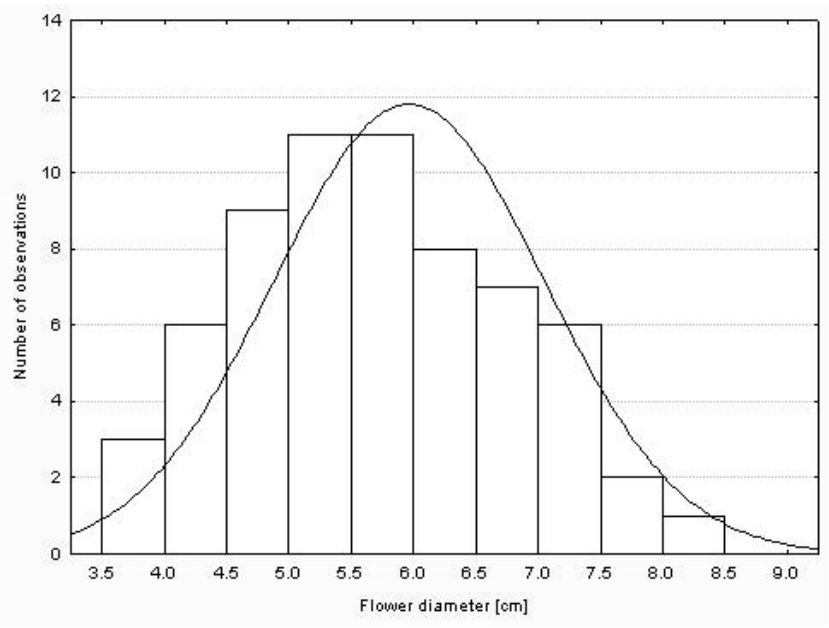

Fig. 5. Flower diameter of $N$. candida specimens.

\section{THE STATE OF THREAT \\ OF NYMPHAEA CANDIDA IN SOUTHERN POLAND}

To show the conservation state of $N$. candida in southern Poland, the number of localities for separate regions were compared (Table 4).

\section{DISCUSSION}

During the presented studies the extent area of southern Poland was surveyed aiming to solve the distribution problem of $N$. candida and N. alba. Both species of water lily are closely related and many morphological features overlap without any distinct differences. However, the number of carpellary teeth and the pollen diameter seems to be the most useful characteristic features for recognition of the two species as it hardly overlaps (Muntendam et al. 1996). The most useful diagnostic feature, even during the field studies, is the number of carpellary teeth, which is countable in flower as well as in the ovary.

Using the current botanical keys we come to the conclusion that, with no doubts, Nymphaea candida occurs in south Poland. Moreover, the preliminary analyses of the rarity of both species show, that $N$. candida is quite frequent. The number of carpellary teeth, which should not exceed 14, was often below 10. The mean value for the examined specimens was 9.29 (from 7 to 12). The mean value of pollen diameter was $43.95 \mu \mathrm{m}$ (the maximum value for $N$. alba doesn't exceed $42 \mu \mathrm{m}$ ) and in some cases even more than $50 \mu \mathrm{m}$ (see the distribution list and Figures 6 and 7). Thus, all the individuals suited the range designated for $N$. candida by Muntendam et al. (1996), Wayda (2000), Rutkowski (1998) and others. Also the other countable features, like the diameter of the flowers (5.95 in average) or the proportion of the stigma diameter to the ovary one (mean value 0.6 ) evidently indicated $N$. candida (Table 1 and 2). Almost all plants had cup-shaped flowers, the 


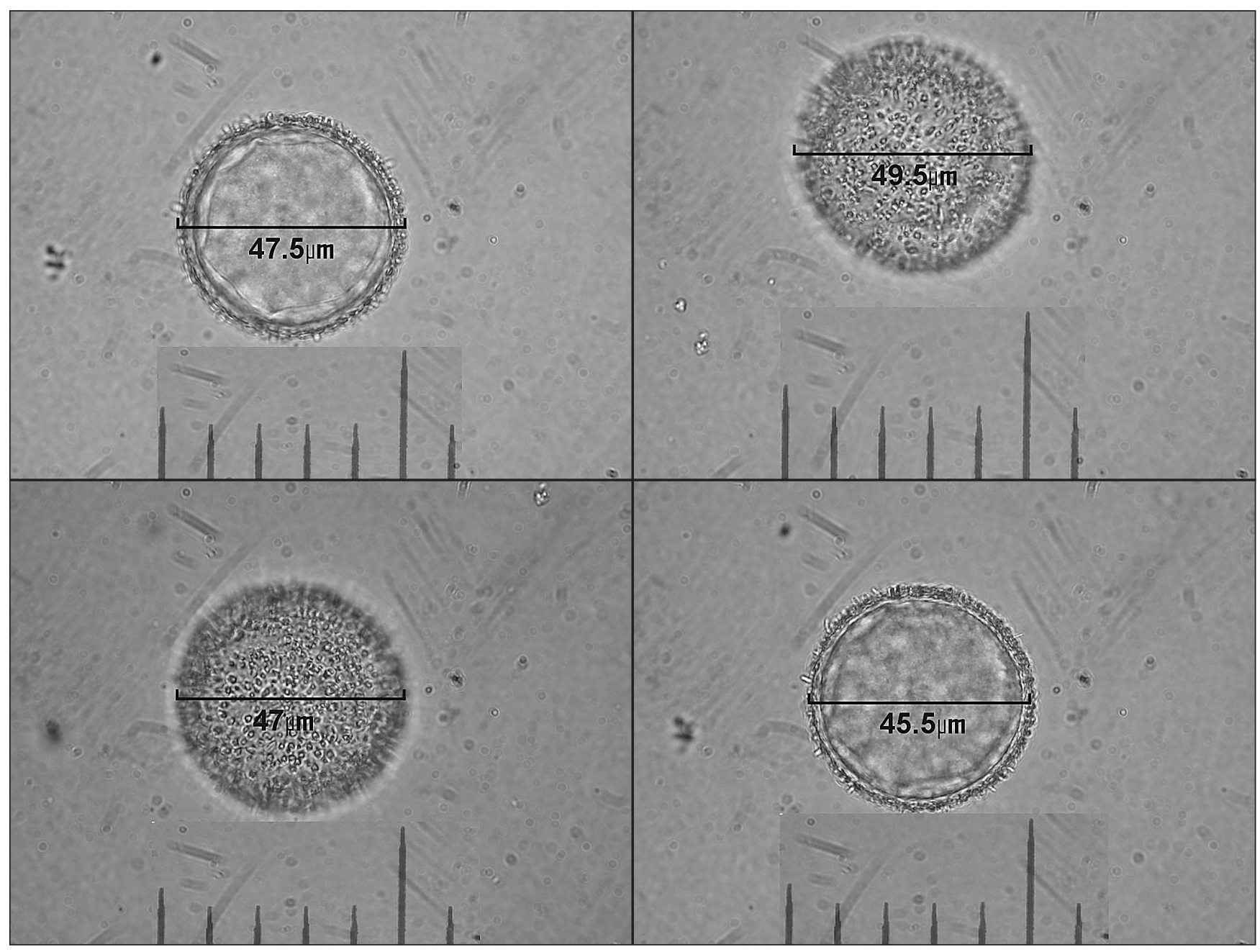

Fig. 6. Morphology of pollens of $N$. candida from Opole Silesia.

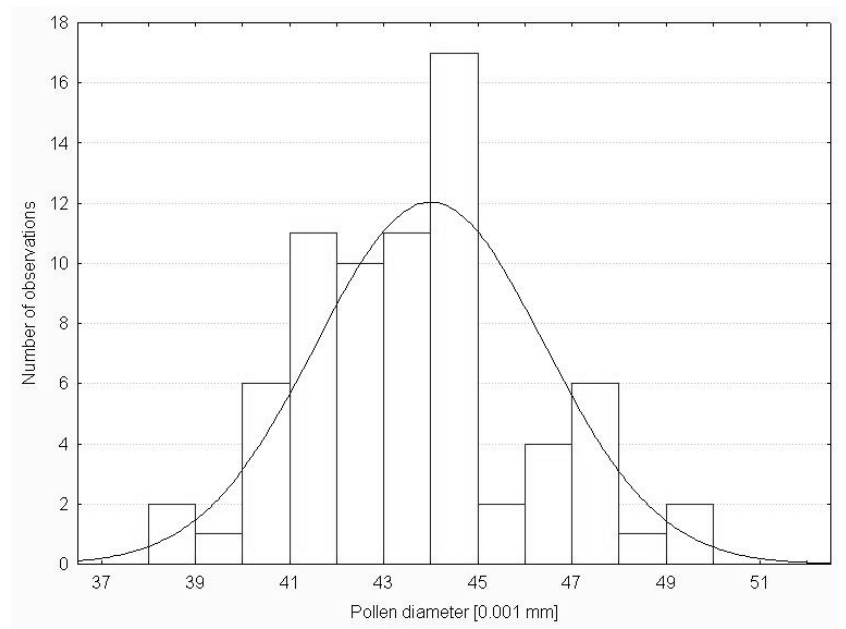

Fig. 7. Pollen diameter of $N$. candida specimens.

square-shaped flowerbase (sometimes with a rough edge), the orange stigma, and the flower partially submerged in the water with erected sepals. Leaves were in the majority red or red-green on the bottom side with converging nerves of leafslips.

The results of the study in comparison to the recent researches on $N$. alba (Nowak and Nowak 2007) revealed the considerable differentiation among Nymphaea species. Remarkable differences were found within some diagnostic

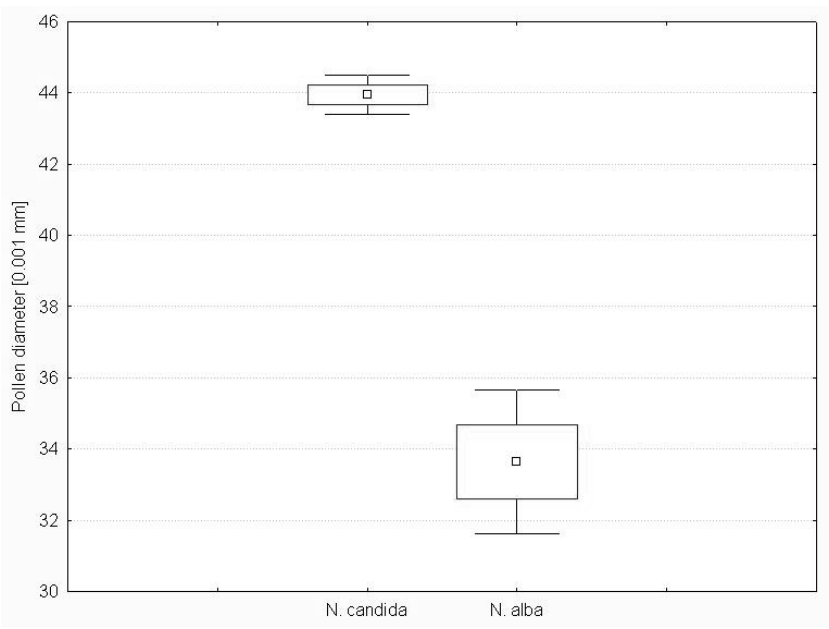

Fig. 8. Results of comparison of pollen diameters for $N$. candida and $N$. alba checked by $t$ test.

features, such as the stigma diameter $(5.95 \mathrm{~mm}$ for $N$. candida and $13.4 \mathrm{~mm}$ for $N$. alba) and the number of carpellary teeth (respectively 9.29 and 16.1). At the same time, the flower diameters also differ significantly reaching 5.95 for $N$. candida and 11.2 for $N$. alba (Table 1). The most important difference is within the value of pollen diameter, which has the average 43.95 for $N$. candida and 33.63 for $N$. alba. These values closely correspond with results of Muntendam et al. (1996) (adequately 43.6 and 34.1). 


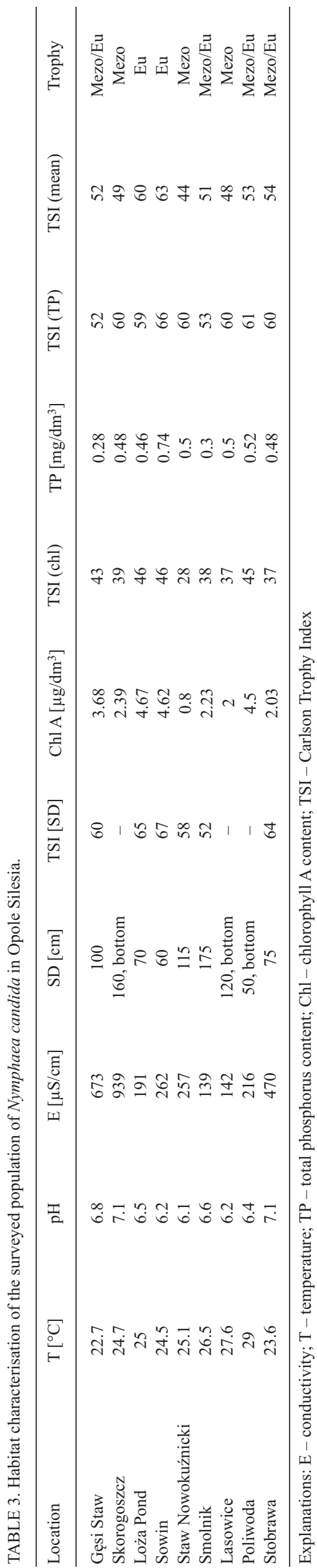

Former habitat analyses show that $N$. candida prefers mesotrophic, clear waters with mineral-organic substrates (Szańkowski and Kłosowski 1999; Kłosowski 2001). Some reports conclude that also eutrophic water reservoirs could give suitable conditions for development of $N$. candida populations (Tomaszewicz 1979). In the Czech Republic N. candida was found in varied types of water, among others in meso- and oligotrophic fish ponds, oxbow lakes and bog ponds (Tomšovic 1997). Within the researched area the species were noted in waters of intermediate character in terms of trophy level, between the values for meso- and eutrophy. Only in three sites populations of $N$. candida were found in evidently mesotrophic waters and in two cases in eutrophic. A slight shifting of the trophy level in the mesotrophy direction for the analysed samples is detectable when investigating the chlorophyll $a$. This analyse classifies the waters generally to mesotrophy and in the case of the Staw Nowokuźnicki pond even to oligotrophy (OECD 1982). Thus, we found the species able to thrive in poor waters of small, mid-forest ponds or bog ponds as well as in rich with mineral substances waters of fish ponds or ox-bow lakes. Such adaptation potential significantly broadened the habitat amplitude of $N$. candida in southern Poland.

However, in comparison to N. alba habitats, it seems to be sure that N. candida occurs in waters of slightly lower trophy level, what was suggested earlier by Tomaszewicz (1979).

The outcomes of the conducted researches have considerable meanings for the phytogeography of the Nymphaea genus in Central Europe. They fill up the distribution gap in the southern part of $N$. candida range in Europe and definitely explain and prove the results of former researches. The continuous distribution of N.candida in southern Poland is also well-founded in climatic and ecological conditions (see Kłosowski 2001). With no doubts the south part of Poland is the transition area where the distribution ranges of both species overlap. Existence of hybrids within the genus could cause the morphological characters very unstable and labile making the easy determination of the species, its hybrids or introgressive forms hardly possible. Further cytological and genetic researches of different specimens of Nymphaea genus in southern Poland should be conducted to solve the problem of genetic flow between the populations of both relatives and their hybrids.

The taxonomic and chorological surveys in neighbouring areas should be attentively monitored. Some interesting new data on the distribution of N. candida were published for the Saxony region in south-east Germany. The revision of the collected specimens and new field studies changed the regional range of the species, considerably diminishing the distribution acreage and splitting it into some small patches (Otto et al. 2000; Otto 2004). Thus, there is a question whether the natural south-western range of $N$. canidida in Germany, Austria, the Czech Republic, Switzerland and Poland is continuous or patchy due to uncomfortable ecological conditions, e.g. too high elevation or a lack of suitable waters. This situation is possible for some areas in south Poland where we found scarce number of the N. candida population (e.g. Małopolska).

\section{THE STATE OF THREAT OF NYMPHAEA CANDIDA IN SOUTHERN POLAND}

The detailed study on the taxonomy and distribution of the species within the Nymphaea genus is obviously indispensable tool in proper management and effective conservation of both taxa (comp. Nowak and Nowak 2004). Without precise data relating to chorology, dynamic and range changes of these plants the legal protection of water lilies could be ineffective.

In the neighbouring countries $N$. candida is considered as a taxon with different dynamic and endangerment status. In the Czech Republic it was categorized as a critically endangered (Procházka 2001). Generally, in the area of Germany it is regarded as an endangered but in Bavaria as a critically endangered (Korneck et al. 1996). In the last edition of the Polish Red Data Book of Plant, Kłosowski (2001) treats $N$. candida as a vulnerable. Surprisingly, the species has not been red listed in Poland (Zarzycki and Szeląg 2006). In separate regions of southern Poland, $N$. candida was evaluated against criteria of IUCN and was classified as a vulnerable taxon in Opole Province (Nowak et al. 2008).

It is hardly possible to precisely determine the real degree of endangerment in the whole country using only informa- 
TABLE 4. The endangerment status of $N$. candida in south Poland.

\begin{tabular}{lccc}
\hline \multirow{2}{*}{ Province } & \multicolumn{2}{c}{ Number of locations } & \\
\cline { 2 - 3 } & Before 2001 & 2001-2008 & \\
\hline Lower Silesian & 34 & 11 & NT \\
Lubelskie & 52 & 5 & EN \\
Małopolskie & 4 & 1 & VU \\
Opolskie & 18 & 27 & LC \\
Podkarpackie & 1 & 2 & VU \\
Silesian & 31 & 4 & EN \\
Świętokrzyskie & 4 & 7 & LC \\
\hline S Poland & 144 & 57 & LC \\
\hline
\end{tabular}

tion from southern regions of Poland. Because of the taxonomical discrepancies there is also a lack of credible data in some regions. So, it seems to be indispensable to verify the synanthropodynamic situation of $N$. cadida as well as N. alba in whole Poland.

The comparative analyses within the different time periods for separate regions of southern Poland show, that for some provinces $N$. candida increases the number of locations. Inconsiderable decrease is observed in case of Lower Silesia, much worse is the situation in Upper Silesia (Table 4). Even worse is the situation in Lubelskie voivodeship, however, there are some additional information relating to pollen findings of $N$. candida in several lakes, which were not included in presented assessment (see Bałaga et al. 2002; Bałaga 2007a, b). In some regions (e.g. Podkarpacie) the species was found for the first time. However it does not prove its expansive properties, but rather reflects the intensification of floristic surveys and shows the potential of $N$. candida to settled in mountainous areas. It is also worth to mention that in some regions (north-eastern part of the Małopolska Uppland or Opole Silesia) the species is more frequent than N. alba (comp. Nobis 2007).

According to the IUCN guidelines to species assessment (IUCN 2001) the data gathered during the presented study do not allow to consider $N$. candida as a vulnerable species in Poland. Still existing populations known from the XIX century, numerous stable locations and abundant populations in some regions at present suggest that the species should be regarded as a least concern (LC). Also the analyze of the habitat accessibility, a biotope extent, an ecological amplitude against the trophy level and direct human impacts support this categorization. Nevertheless it is probable, that in some regions the species could have the considerable withdrawing tendency and has to be red listed in higher categories.

\section{ACKNOWLEDGEMENTS}

The authors want to thank Prof. Wiesław Włoch (Polish Academy of Sciences, Katowice) for the assistance in measuring the pollen diameter.

\section{LITERATURE CITED}

ANIOŁ-KWIATKOWSKA J., BERDOWSKI W., KOŁA W., KWIATKOWSKI P., MACICKA T., PANEK E., PENDER K., WERETELNIK E., WILCZYŃSKA W. 1995. Charakterystyka botaniczna rezerwatu "Stawy Milickie". Acta Univ. Wrat. Prace Bot. 62: 199-233. (in Polish)
ANIOŁ-KWIATKOWSKA J., DAJDOK Z., KĄCKI Z. 1998. Walory przyrodnicze projektowanego Parku Krajobrazowego "Dolina Odry II". Acta Univ. Wrat. Prace Bot. 74: 201-233. (in Polish)

BAŁAGA K. 2007a. Changes in the natural environment recorded in the sediments of the Karaśne Lake-mire complex (Lublin Polesie, E Poland). Geochronometria 29: 1-21.

BAŁAGA K. 2007b. Transformation of lake ecosystem into peat bog and vegetation history based on Durne Bagno mire (Lublin Polesie, E Poland). Geochronometria 29: 23-43.

BAŁAGA K., SZEROCZYŃSKA K., TARAS H., MAGIERSKI J. 2002. Natural and anthropogenic conditioning of the development of Lake Perespilno (Lublin Polesie). Limnological Review 2, 15-27.

BARBER E. 1937. Flora der Oberlausitz einschließlich den nördlichen Böhmens. Abhandlungen der Naturforschenden Gesellschaft zu Görlitz 33(1): 7-88.

BENKERT D., FUKAREK F., KORSCH H. 1996. Verbreitungsatlas der Farn- und Blütenpflanzen Ostdeutschlands (Meklemburg-Vorpommern, Brandenburg, Berlin, SachsenAnhalt, Sachsen, Thüringen). Gustav Fischer Jena, Stuttgart, Lübeck, Ulm, pp. 55.

BERDOWSKI W., NARKIEWICZ C. 1996. Inwentaryzacja stanowisk chronionych gatunków roślin i grzybów na terenie gminy Węgliniec. Jelenia Góra-Wrocław (unpublished work).

BOBROWICZ G., KONIECZNY K. 2000. Osobliwości przyrodnicze gminy Wińsko i propozycja ich ochrony. Chrońmy Przyr. Ojcz. 64(4): 5-31.

CARLSON R.E. 1977. A trophic state index for lakes. Limnol. Oceanogr. 22: 361-369.

CASPER S.J., KRAUSCH H.-D. 1981. Süßwasserflora von Mitteleuropa. Band 24, pp. 461-465. Gustav Fischer Verlag, Stuttgart, New York.

CELIŃSKI F., ROSTAŃSKI K., SENDEK A., WIKA S., CABAŁA S. 1979. Nowe stanowiska rzadkich roślin na Górnym Śląsku i terenach przyległych. Cz. IV. Zesz. Przyr. OTPN 18: 3-18.

CIEŚLAK M., SZLACHETKA A. 1987. Ekspertyza botaniczna zachodniej części województwa legnickiego. Na zlecenie Woj. Konserwatora Przyrody w Legnicy (unpublished work).

CONARD H.S. 1905. The waterlilies. A monograph of the genus Nymphaea. The Carnegie Institution of Washington. Publ. 4: 172-179.

CZARNECKA B., SUGIER P. 1998. Landscape changes in macro- and microscales. Ekológia (Bratislava), 17, suppl. I: 177-188.

DAJDOK Z., KĄCKI Z., NOWAK A., NOWAK S., SPAŁEK K. 1998. Atlas rozmieszczenia roślin naczyniowych prawnie chronionych w województwie opolskim. Uniwersytet Opolski, Opole. pp. 278.

DENISIUK Z., GRYNIA M. 1969. Mszar sfagnowy Rhynchosporetum albae Koch 1926 w dolinie górnej Warty. Fragm. Flor. Geobot. 15(3): 343-351.

DODDS W.K., JONES J.R., WELCH E.B. 1998. Suggested classification of stream trophic state: distribution of temperate stream types by chlorophyll, total nitrogen, and phosphorus. Wat. Res. 32(5): 1455-1462.

FIEK E. 1881. Flora von Schlesien preussischen und österreichischen Antheils, enthaltend die wildwachsenden, verwilderten und angebauten Phanerogamen und Gefäss-Cryptogramen. J. U. Kern's Verlag, Breslau, pp. 571.

FIEK E. 1887. Resultate der Durchforschung der schlesischen Phanerogamenflora im Jahre 1886. Jahr. Ber. Schles. Gesell. vaterl. Cultur 64: 197-224.

FIEK E. 1888. Resultate der Durchforschung der schlesischen Phanerogamenflora im Jahre 1887. Jahr. Ber. Schles. Gesell. vaterl. Cultur 65: 309-339.

FIEK E., SCHUBE T. 1893. Ergebnisse der Durchforschung der schlesischen Phanerogamenflora im Jahre 1892. Jahr. Ber. Schles. Gesell. vaterl. Cultur 70: 84-108. 
FIJAŁKOWSKI D. 1959a. Szata roślinna jezior Łęczyńsko-Włodawskich i przylegających do nich torfowisk. Ann. Univ. Mariae Curie-Skłodowska, sec. B, 14(3): 131-206.

FIJAŁKOWSKI D. 1959b. Wykaz rzadszych roślin Lubelszczyzny. Cz. III. Fragm. Flor et Geobot. 5(1): 11-35.

FIJAŁKOWSKI D. 1960. Wykaz rzadszych roślin Lubelszczyzny. Cz. IV. Fragm. Flor et Geobot. 6(3): 262-286.

FIJAŁKOWSKI D. 1961. Zbiorowiska roślinne jeziora Bartków w woj. lubelskim. Ann. Univ. Mariae Curie-Skłodowska, sec. C, 16(4): 77-89.

FIJAŁKOWSKI D. 1962. Wykaz rzadszych roślin Lubelszczyzny. Cz. V. Fragm. Flor et Geobot. 8(4): 443-468.

FIJAŁKOWSKI D. 1963. Wykaz rzadszych roślin Lubelszczyzny. Cz. VI. Fragm. Flor et Geobot. 9(2): 219-237.

FIJAŁKOWSKI D. 1964. Wykaz rzadszych roślin Lubelszczyzny. Cz. VII. Fragm. Flor et Geobot. 10(4): 453-471.

FIJAŁKOWSKI D. 1965. Zbiorowiska wodno-torfowiskowe rezerwatu Świerszczów koło Włodawy. Annales UMCS, sec. C, 20(12): 179-195.

FIJAŁKOWSKI D. 1997. Szata roślinna Parku Krajobrazowego "Lasy Janowskie". Wyd. UMCS, Lublin.

FIJAŁKOWSKI D., BLOCH M., FLISIŃSKA Z., POLSKI A., WÓJCIAK H. 1992. Szata roślinna rezerwatu Imielty Ług. Ann. Univ. Mariae Curie-Skłodowska, sec. C, 47: 169-197.

FIJAŁKOWSKI D., WAWER M., PIETRAS T. 1993. Roślinność projektowanego rezerwatu Brudno na Pojezierzu ŁęczyńskoWłodawskim. Ann. Univ. Mariae Curie-Skłodowska, sec. C, 48: 93-103.

FIJAŁKOWSKI D., KARCZMARZ K., WÓLCZYŃSKA R. 1994a. Zmiany szaty roślinnej bagna Dubeczyńskiego w okresie 35 lat (1958-1993). Ann. Univ. Mariae Curie-Skłodowska, sec. C, 49: 101-132.

FIJAŁKOWSKI D., WAWER M., PIETRAS T. 1994b. Roślinność rezerwatu Żółwiowe Błota koło Włodawy. Ann. Univ. Mariae Curie-Skłodowska, sec. C, 49: 151-170.

FOJCIK B. 1997. Rośliny chronione Wyżyny Wieluńskiej. Acta Biol. Silesiana 30(47): 125-138.

FOJCIK B. 2003. Osobliwości szaty roślinnej okolic Myszkowa i problemy ich ochrony, Chrońmy Przyr. Ojcz. 9(1): 94-98.

GLÜCK H. 1924. Biologische und Morfologische Untersuchungen über Wasser- und Sumpfgewächse, IV Teil. Untergetauchte und Schwimmblattflora, pp. 328-459. Gustav Fischer Verlag, Jena.

HEGI E. 1965. Familie Nymhaeaceae. Illustrierte Flora von Mitteleuropa. Band III, pp. 1-29. Carl Hansen Verlag, Wien.

HEJNÝ S., SLAVÍK B. 1997. Květena České Republiky. Vol. 1. Academia Praha, pp. 557.

HEREŹNIAK J. 1986. Nowe stanowiska rzadkich i interesujących gatunków roślin naczyniowych w północnej części Wyżyny Śląsko-Krakowskiej. Fragm. Flor. Geobot. 29(3-4): 361-384.

HEREŹNIAK J. 1993. Stosunki geobotaniczno-leśne północnej części Wyżyny Śląsko-Krakowskiej na tle zróżnicowania i przemian środowiska. Monographiae Botanice, pp. 75.

HEREŹNIAK J., GRZYL A., KOŁODZIEJEK J., ŁAWRYNOWICZ M. 2001, Materiały do flory północnej części Wyżyny Śląsko-Krakowskiej - rzadkie i interesujące gatunki roślin naczyniowych. Cz. 2. Fragm. Flor. Geobot. Polonica 8: 35-41.

HEREŹNIAK J., SAMOSIEJ L. 1990. O potrzebie ochrony rezerwatowej Jeziora Blachownia koło Częstochowy, Chrońmy Przyr. Ojcz. 46(1): 35-46.

HEUKELS H., VAN DER MEIJDEN R. 1990. Flora von Nederland. Wolters-Noordhoff, Groningen, pp. 144.

HULTEN E., FRIES M. 1986. Atlas of North European vascular plants. North of the tropic of cancer. Vol. 1. Koeltz Scientific Books, Köenigstein, 498 pp.

IUCN. 2001. IUCN Red List Categories and Criteria: Version 3.1. IUCN Species Survival Commission. IUCN, Gland, Switzerland and Cambridge, UK. ii +30 .
JONES M. R., CLARKE G. C. S. 1981. Nymphaeaceae. In: Punt W., Clarke G.C.S. (eds), The Northwest European Pollen Flora. III, pp. 56-67. Elsevier Scientific Publishing Comp., Amserdam, Oxford, New York.

KARCZMARZ K., MALICKI J. 1971. Zespoły i ekologia ramienic Pojezierza Łęczyńsko-Włodawskiego. Ann. Univ. Mariae Curie-Skłodowska, Lublin, sec. C, 26(23): 297-327.

KŁOSOWSKI S. 2001. Nymphaea candida C. Presl. Grzybienie północne. pp. 113-116. In: Kaźmierczakowa R., Zarzycki K. (eds), Polska czerwona księga roślin. Paprotniki i rośliny kwiatowe. Instytut Botaniki im. W. Szafera, Instytut Ochrony Przyrody PAN. Kraków, pp. 664.

KORNECK D., SCHNITTLER M., VOLLMER I. 1996. Rote Liste der Farn- und Blütenpflanzen (Pteridophyta et Spermatophyta) Deutschlands. pp. 21-187. In: Ludwig G., Schnittler M. (eds), Rote Liste gefährdeter Pflanzen Deutschlands. Schr.-R. f. Vegetationskunde 28. Bundesamt für Naturschutz, BonnBad Godesberg. pp. 744+VXI.

KUBÁT K. 2002. Klič ke květeně České republiky. Academia Praha, pp. 927.

KUCZYŃSKA I. 1974: Stosunki geobotaniczne Opolszczyzny. I. Zbiorowiska leśne. Acta Univ. Wrat. 162, Prace Bot. 18, pp. 115.

KUJAWA-PAWLACZYK J., PAWLACZYK P. 2003. Ochrona rzadkich i zagrożonych roślin w lasach. Dodatek 2 - Cenne gatunki roślin na terenie Regionalnej Dyrekcji Lasów Państwowych w Zielonej Górze. Wyd. Klubu Przyrodników, Świebodzin, pp. 142.

KUŹNIEWSKI E. 1964. Notatki florystyczne ze Śląska. Cz. IV, Zesz. Przyr. OTPN 4: 79-83.

MACICKA T., WILCZYŃSKA W. 1992. Inwentaryzacja stanowisk roślin chronionych na terenie gminy Wińsko. Maszynopis. Dolnośląski Urząd Wojew. we Wrocławiu, Woj. Kons. Przyr.

MACICKA-PAWLIK T., WILCZYŃSKA W. 1996. Zbiorowiska roślinne starorzeczy w dolinie środkowego biegu Odry. Acta Univ. Wrat. No 1735, Prace Bot. 64: 74-120.

MACICKA-PAWLIK T., WILCZYŃSKA W. 1998. Wartości przyrodnicze projektowanego Parku Krajobrazowego "Dolina Odry I". Acta Univ. Wrat. Prace Bot. 74: 165-200.

MEUSEL H., JÄGER E., WEINERT E. 1965. Vergleichende Chorologie der Zentraleuropäischen Flora. 1 Karten. Gustav Fischer Verlag, Jena, pp. 258.

MUNTENDAM J.B., POVEL G.D.E., VAN DER VELDE G. 1996. Morphometric patterns in the Nymphaea alba-candida complex. Acta. Bot. Neerl. 45(3): 279-302.

MICHALAK S. 1963. Staw Nowokuźnicki - rezerwat na Śląsku Opolskim. Chrońmy Przyr. Ojcz. 19(6): 24-27.

NEUHÄUSL R., TOMŠOVIC P. 1957. Die Gattung Nymphaea (L.) Smith in der Tschechoslovakei. Preslia 29: 225-249.

NOBIS M. 2007. Rośliny naczyniowe zachodniej części Przedgórza Iłżeckiego. Vascular plants of the west part of Iłża Foreland (Małopolska Upland). Prace Bot. 40: 1-458. (in Polish with English summary)

NOWAK A., NOWAK S. 2004. The effectiveness of plant conservation: a case study of Opole Province, Southwest Poland. Environmental Management, Springer-New York, Vol. 34(3): 363-371.

NOWAK A., NOWAK S. 2007. The problem of the occurrence of Nymphaea candida C. Presl in the Opole Silesia. Nat. J. 40: 25-33.

NOWAK A., NOWAK S., SPAŁEK K. 2008. Red list of vascular plants of Opole province - 2008. Opol. Scient. Soc., Nat. J. 41: 141-158.

OBERDORFER E. 1994. Pflanzensoziologische Exkursionsflora. 7 Auflage. Verlag Eugen Ulmer, Stuttgart. 1050 pp.

OCHYRA R. 1979. Flora lejków krasowych w okolicach Staszowa. I. Rośliny naczyniowe. Fragm. Flor. Geobot. Polonica 25: 209-236

OECD 1982. Organization for Economic Co-operation and Development. Eutrophication of waters: monitoring, assessment and control. OECD, Paris, pp. 154. 
OTTO H.-W. 2004. Die Farn- und Samenpflanzen der Oberlausitz. Berichte der Naturforschenden Gesellschaft der Oberlausitz 12: 63 .

OTTO H.-W., GEBAUER P., HARDTKE H.-J. 2000. Floristische Beobachtungen 1999 in Oberlausitz und Elbhügelland. Berichte der Naturforschenden Gesellschaft der Oberlausitz 9: 135-147.

PARINET B., LHOTE A., LEGUBE B. 2004. Principal component analysis: an appropriate tool for water quality evaluation and management - application to a tropical lake system. Ecological Modelling 178: 295-311.

PEŁECHATY M., PEŁECHATA A., PUKACZ A. 2007. Charophyte flora and vegetation against the background of the trophy state of lakes of Lubuskie Lakeland (mid-Western Poland). Bogucki Publisher, Poznań, pp. 137 (in Polish)

POPIOŁEK Z. 1971. Roślinność wodna i przybrzeżna jezior okolic Ostrowa Lubelskiego na tle warunków siedliskowych. Cz. I. Jezioro Kleszczów. Ann. Univ. Mariae Curie-Skłodowska, sec. C, 26: 387-408

POPIOŁEK Z. 1973. Roślinność wodna i przybrzeżna jezior okolic Ostrowa Lubelskiego na tle warunków siedliskowych. Cz. III. Jezioro Miejskie. Ann. Univ. Mariae Curie-Skłodowska, sec. C, 28: 191-213.

POPIOŁEK Z. 1974. Roślinność wodna i przybrzeżna jezior okolic Ostrowa Lubelskiego na tle warunków siedliskowych. Cz. IV. Jezioro Czarne Gościnieckie. Ann. Univ. Mariae CurieSkłodowska, sec. C, 29: 333-353.

POPIOŁEK Z. 1988. Zróżnicowanie roślinności wodnej i przybrzeżnej na tle warunków siedliskowych w obrębie kompleksu jezior Lubelskiego Zagłębia Węglowego. Wyd UMCS, 35: 1-108.

PROCHÁZKA F. (ed.). 2001. Černý a červený seznam cévnatých rostlin České republiky (stav v roce 2000). Př́roda, Praha 18: 1-166.

RADICS F. 1967. A revision of the Nymphaea material in Hungarian Natural History Museum. Pars Bot. 59: 135-145.

ROZPORZĄDZENIE MINISTRA ŚRODOWISKA 2004. Rozporządzenie Ministra Środowiska z dnia 9 lipca 2004 r. w sprawie gatunków dziko występujących roślin objętych ochroną. Dz. U. Nr 168, poz. 1764.

RUSZLEWICZ A., SZLACHETKA A. 2007. Ocena oddziaływania na obszary Natura 2000 obejście Ścinawy z przeprawą mostową na Odrze (unpublished work).

RUTKOWSKI L. 1998. Klucz do oznaczania roślin naczyniowych Polski niżowej. Wydawnictwo Naukowe PWN, Warszawa, pp. 812.

SCHALOW E. 1935. Ergebnisse der Durchforschung der schlesischen Gefässpflanzenwelt im Jahre 1934. Jahresber. Schles. Ges. vaterl. Cult.107: 55-70.

SCHUBE T. 1903a. Die Verbreitung der Gefässpflanzen in Schlesien, preussischen und österreichischen Anteils. Druck von R. Nischowsky, Breslau, pp. 361.

SCHUBE T. 1903b. Ergebnisse der Durchforschung der schlesischen Gefässpflanzenwelt im Jahre 1902. Jahr. Ber. Schles. Gesell. vaterl. Cultur 80: 33-59.

SCHUBE T. 1904. Ergebnisse der Durchforschung der schlesischen Gefässpflanzenwelt im Jahre 1903. Jahr. Ber. Schles. Gesell. vaterl. Cultur 81: 42-64.

SCHUBE T. 1905. Ergebnisse der Durchforschung der schlesischen Gefässpflanzenwelt im Jahre 1904. Jahresber. Schles. Ges. vaterl. Cult. 82: 41-64

SCHUBE T. 1906. Ergebnisse der Durchforschung der schlesischen Gefässpflanzenwelt im Jahre 1905. Jahr. Ber. Schles. Gesell. vaterl. Cultur 83: 75-95.

SCHUBE T. 1908. Ergebnisse der Durchforschung der schlesischen Gefässpflanzenwelt im Jahre 1907. Jahresber. Schles. Ges. vaterl. Cult. 85: 46-62.

SCHUBE T. 1910. Ergebnisse der Durchforschung der schlesischen Gefässpflanzenwelt im Jahre 1909. Jahresber. Schles. Ges. vaterl. Cult. 87: 49-73.
SCHUBE T. 1925. Ergebnisse der Durchforschung der schlesischen Gefässpflanzenwelt im Jahre 1919-1924. Jahresber. Schles. Ges. vaterl. Cult. 97: 75-81.

SENDEK A. 1984. Rośliny Naczyniowe Górnośląskiego Okręgu Przemysłowego. OTPN, PWN, Warszawa-Wrocław. 137 pp.

SENDEK A. 1986. Charakterystyka flory rezerwatu Łężczak koło Raciborza. Zesz. Przyr. OTPN 24: 9-19.

SOLHEIM L. A. 2005. Reference conditions of European lakes. Draft version, pp. 105.

SPAŁEK K. 2007. Nymphaeetum albae Vollmar 1947 em. Oberd. in Oberd. et al. 1967, a plant association new to Poland, Acta Soc. Bot. Pol. 76(1): 81-84.

SUGIER P. 1998. Przekształcenia szaty roślinnej jeziora Czarne Gościnieckie na Pojezierzu Łęczyńsko-Włodawskim. Przegląd Przyrodniczy, 9(1/2): 213-222.

SUGIER P., CZARNECKA B. 1998. Changes of geocomponents in the landscape of the Polesie Lubelskie under the influence of anthropopression. In: Richling A., Lechnio J., Malinowska E. (eds), Landscape transformation in Europe - practical and theoretical aspects. The problems of landscape ecology 3: 236-245.

SUGIER P., POPIOŁEK Z. 1995. Roślinność wodna i przybrzeżna jezior Poleskiego Parku Narodowego na tle warunków siedliskowych. Jezioro Karaśne. Ann. Univ. Mariae Curie-Skłodowska, sec. C, 50(3): 55-69.

SUGIER P., POPIOŁEK Z. 1998. Roślinność wodna i przybrzeżna jeziora Moszne w Poleskim Parku Narodowym. Ann. Univ. Mariae Curie-Skłodowska, sectio C, 53: 185-200.

SZAFRAN B. 1925. Der Bau und das Alter des Moores von Pakosław bei Iłża in Mittelpolen. Bull. Acad. Pol. Sci. Lettr. Cl. Math.-Nat., Ser. B 7: 751-768.

SZAŃKOWSKI M., KŁOSOWSKI S. 1999. Habitat conditions of nymphaeid associations in Poland. Hydrobiologia 415: 177-185 .

SZLACHETKA A. 2007a. Weryfikacja siedlisk leśnych i nieleśnych w Nadleśnictwie Chocianów, w ramach inwentaryzacji siedlisk Natura 2000 w Lasach Państwowych (unpublished work).

SZLACHETKA A. 2007b. Weryfikacja siedlisk leśnych w Nadleśnictwie Legnica, w ramach inwentaryzacji siedlisk Natura 2000 w Lasach Państwowych (unpublished work).

SZLACHETKA A. 2008. Ekspertyza dotycząca projektu obszarów Gałuszka w Chocianowie, Wzgórza Warzęgowskie, Wzgórza Dalkowskie sieci Natura 2000 w województwie dolnośląskim. Na zlecenie Ministra Środowiska (unpublished work).

SZLACHETKA A., SZLACHETKA E. 1998. Dokumentacja projektowanego rezerwatu przyrody "Torfowisko Borówki". Na zlecenie Woj. Konserwatora Przyrody w Legnicy (unpublished work).

TOMA C. 1994. Czy kotewka orzech wodny Trapa natans powróci na dawne stanowisko w zbiorniku wodnym Paprocany (Tychy)? Chrońmy Przyr. Ojcz. 50(1): 84-87.

TOMASZEWICZ H. 1979. Roślinność wodna i szuwarowa Polski. Rozprawy Uniwersytetu Warszawskiego, Warszawa, pp. 325.

TOMŠOVIC P. 1997. Nymphaeaceae Salisb. pp. 325-363. In: Hejný S., Slavík B. (eds), Květena České Republiky. Vol. 1. Academia, Praha, pp. 557.

TUTIN T.G., BURGES N.A., CHATER A.O., EDMONDSON J.R., HEYWOOD V.H., MOORE D.M., VALENTINE D.H., WALTERS S.M., WEBB D.A. 2002. Flora Europaea. Vol. 1, Psilotaceae to Platanaceae. Cambridge University Press. 581 pp.

UECHTRITZ R. VON. 1864a. Beiträge zur Flora von Schlesien. I. Ueber die Flora der Umgegend von Koschentin. Jahresber. Schles. Ges. Vaterl. Cultur, Abhandl. Schles. Ges. Vaterl. Cultur Abt. Naturw. Med. 1: 16-21.

UECHTRITZ R. VON. 1864b. Beiträge zur Flora von Schlesien. II. Seltenere Pflanzen der Flora von Rybnik. Jahresber. Schles. Ges. Vaterl. Cultur, Abhandl. Schles. Ges. Vaterl. Cultur Abt. Naturw. Med. 1: 22-26. 
UECHTRITZ R. VON. 1885. Resultate der Durchforschung der schlesischen Phanerogamenflora im Jahre 1884. Jahr. Ber. Schles. Gesell. vaterl. Cultur 26: 309-341.

URBISZ A. 1996. Flora naczyniowa Płaskowyżu Rybnickiego na tle antropogenicznych przemian tego obszaru. Scripta Rudensia 6: 1-173.

URBISZ A., URBISZ A. 1998. Rośliny chronione południowozachodniej części Wyżyny Śląskiej. Acta Biol. Silesiana 30(47): 113-141.

WAYDA M. 2000. The distribution of Nymphaea candida C. Presl. (Nymphaeceae) in Poland. Acta Soc. Bot. Pol. 69(1): 75-78.

WIMMER F. 1844: Flora von Schlesien. Verl. von F. Hirt. Breslau. pp. 792.
ZABAWSKI J., MATUŁA J. 1973. Grzybienie północne Nymphaea candida w powiecie Zgorzeleckim na Dolnym Śląsku. Chrońmy Przyr. Ojcz. 29 (1): 76-80.

ZAJĄC A. 1978. Założenia metodyczne "Atlasu rozmieszczenia roślin naczyniowych w Polsce”. Wiad. Bot. 22(3): 145-155.

ZAJĄC A., ZAJĄC M. (eds). 2001. Atlas rozmieszczenia roślin naczyniowych w Polsce. Nakładem Pracowni Chorologii Komputerowej Instytutu Botaniki Uniwersytetu Jagiellońskiego. Kraków, pp. 715.

ZARZYCKI K., SZELACG Z. 2006. Red list of the vascular plants in Poland, pp. 9-20. In: Mirek Z., Zarzycki K., Wojewoda W., Szeląg Z. (eds), Red list of plants and fungi in Poland. W. Szafer Institute of Botany, Polish Academy of Sciences, Kraków, pp. 99. 